Relations of Borehole Resistivity to the Horizontal Hydraulic

Conductivity and Dissolved-Solids

Concentration in Water of Clastic

Coastal Plain Aquifers in the

Southeastern United States

United States

Geological

Survey

Water-Supply

Paper 2414

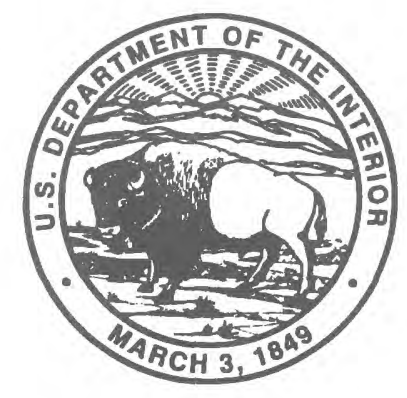


Instructions on ordering publications of the U.S. Geological Survey, along with prices of the last offerings, are given in the current-year issues of the monthly catalog "New Publications of the U.S. Geological Survey." Prices of available U.S. Geological Survey publications released prior to the current year are listed in the most recent annual "Price and Availability List." Publications that may be listed in various U.S. Geological Survey catalogs (see back inside cover) but not listed in the most recent annual "Price and Availability List" may be no longer available.

Reports released through the NTIS may be obtained by writing to the National Technical Information Service, U.S. Department of Commerce, Springfield, VA 22161; please include NTIS report number with inquiry.

Order U.S. Geological Survey publications by mail or over the counter from the offices given below.

\section{BY MAIL}

\section{Books}

Professional Papers, Bulletins, Water-Supply Papers, Techniques of Water-Resources Investigations, Circulars, publications of general interest (such as leaflets, pamphlets, booklets), single copies of Earthquakes \& Volcanoes, Preliminary Determination of Epicenters, and some miscellaneous reports, including some of the foregoing series that have gone out of print at the Superintendent of Documents, are obtainable by mail from

\section{U.S. Geological Survey, Map Distribution Box 25286, MS 306, Federal Center Denver, CO 80225}

Subscriptions to periodicals (Earthquakes \& Volcanoes and Preliminary Determination of Epicenters) can be obtained ONLY from the

\section{Superintendent of Documents \\ Government Printing Office \\ Washington, D.C. 20402}

(Check or money order must be payable to Superintendent of Documents.)

\section{Maps}

For maps, address mail orders to

\section{U.S. Geological Survey, Map Distribution Box 25286, Bldg. 810, Federal Center Denver, CO 80225}

Residents of Alaska may order maps from

U.S. Geological Survey, Earth Science Information Center 101 Twelfth Ave. - Box 12

Fairbanks, AK 99701

\section{OVER THE COUNTER}

\section{Books and Maps}

Books and maps of the U.S. Geological Survey are available over the counter at the following U.S. Geological Survey offices, all of which are authorized agents of the Superintendent of Documents:

- ANCHORAGE, Alaska-Rm. 101, 4230 University Dr.

- LAKEWOOD, Colorado-Federal Center, Bldg. 810

- MENLO PARK, California-Bldg. 3, Rm. 3128, 345 Middlefield Rd.

- RESTON, Virginia-USGS National Center, Rm. 1C402, 12201 Sunrise Valley Dr.

- SALT LAKE CITY, Utah-Federal Bldg., Rm. 8105, 125 South State St.

- SPOKANE, Washington-U.S. Post Office Bldg., Rm. 135, West 904 Riverside Ave.

- WASHINGTON, D.C.-Main Interior Bldg., Rm. 2650, 18th and C Sts., NW.

\section{Maps Only}

"Maps may be purchased over the counter at the following U.S. Geological Survey offices:

- FAIRBANKS, Alaska-New Federal Bldg., 101 Twelfth Ave.

- ROLLA, Missouri-1400 Independence Rd.

- STENNIS SPACE CENTER, Mississippi-Bldg. 3101 
Relations of Borehole Resistivity to the Horizontal Hydraulic Conductivity and Dissolved-Solids Concentration in Water of Clastic Coastal Plain Aquifers in the Southeastern United States

By ROBERT E. FAYE and WINSTON G. SMITH 


\section{U.S. DEPARTMENT OF THE INTERIOR BRUCE BABBITT, Secretary}

\section{U.S. GEOLOGICAL SURVEY}

GORDON P. EATON, Director

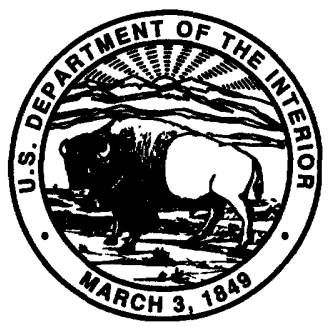

Any use of trade, product, or firm names

in this publication is for descriptive purposes only

and does not imply endorsement by the U.S. Government.

For sale by

U.S. Geological Survey, Map Distribution

Box 25286, MS 306, Federal Center

Denver, CO 80225

\section{Library of Congress Cataloging in Publication Data}

Faye, Robert E.

Relations of borehole resistivity to the horizontal hydraulic conductivity and dissolved-solids concentration in water of clastic coastal plain aquifers in the southeastern United States / by Robert E. Faye and Winston G. Smith. p. $\quad$ cm. - (U.S. Geological Survey water-supply paper ; 2414)

Includes bibliographical references.

1. Groundwater flow-Southern States-Measurement. 2. Geophysical well logging-Southern States. 3. Aquifers-Southern States. 4. Water quality-Southern States. I. Smith, Winston G. II. Title. III. Series. GB1197.7.F38 1994

$551.49^{\prime} 0975-\mathrm{dc} 20$ 


\section{CONTENTS}

Introduction $\ldots \ldots \ldots \ldots \ldots$

\section{FIGURES}

1. Map showing study area and county and site locations of wells

2-9. Graphs showing:

2. Resistivity borehole log at site $\mathrm{CH}-186$ showing short- and long-normal curves and the screened and estimated contributing intervals.

3. Relation of horizontal hydraulic conductivity to grain-surface resistivity as defined by the summation

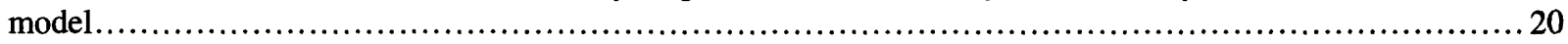

4. Relation of horizontal hydraulic conductivity to apparent formation factor as defined by the product

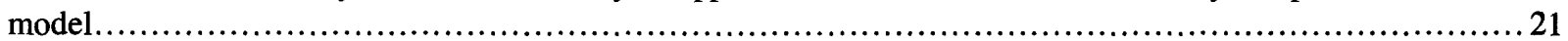

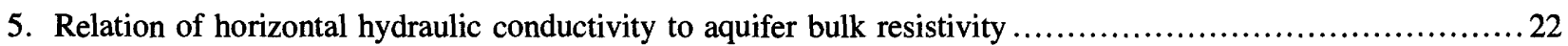

6. Relation of horizontal hydraulic conductivity to aquifer water resistivity ................................23

7. Relation of dissolved-solids concentration in aquifer water to aquifer water resistivity .....................24

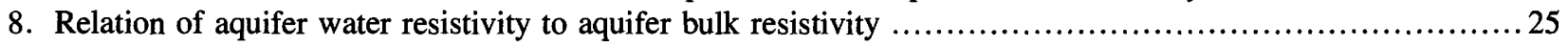

9. Relation of dissolved-solids concentration in aquifer water to aquifer bulk resistivity ........................26

\section{TABLES}

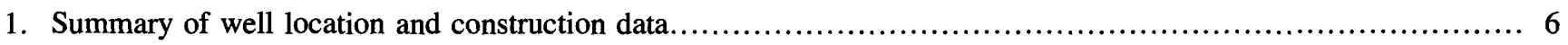

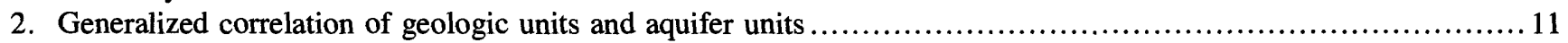

3. Summary of borehole geoelectrical properties and aquifer water-quality characteristics at well sites ..............12

4. Summary of borehole geoelectrical properties and aquifer hydraulic characteristics at well sites ...................16

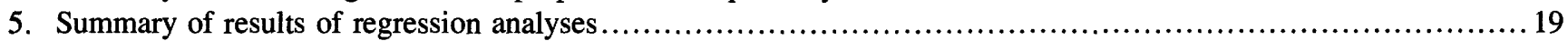

6. Summary of regression model estimates of aquifer water-quality characteristics ..............................2 27

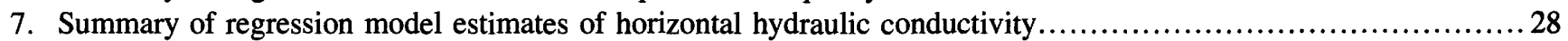

8. Summary of error analyses of regression model estimates of aquifer water-quality characteristics ...................30

9. Summary of error analyses of regression model estimates of horizontal hydraulic conductivity ...................3 30 
CONVERSION FACTORS

\begin{tabular}{rll} 
Muitiply & By & To obtain \\
\hline inch (in.) & 25.4 & millimeter $(\mathrm{mm})$ \\
foot $(\mathrm{ft})$ & 0.3048 & meter $(\mathrm{m})$ \\
mile $(\mathrm{mi})$ & 1.609 & kilometer $(\mathrm{km})$ \\
square mile $\left(\mathrm{mi}^{2}\right)$ & 2.590 & square kilometer $\left(\mathrm{km}^{2}\right)$ \\
square foot per day $\left(\mathrm{ft}^{2} / \mathrm{d}\right)$ & 0.09290 & square meter per day $\left(\mathrm{m}^{2} / \mathrm{d}\right)$ \\
cubic foot per second $\left(\mathrm{ft}^{3} / \mathrm{s}\right)$ & 0.02832 & cubic meter per second $\left(\mathrm{m}^{3} / \mathrm{s}\right)$ \\
cubic foot per second & 0.01093 & cubic meter per second \\
per square mile $\left(\mathrm{m}^{3} / \mathrm{s} / \mathrm{km}^{2}\right)$ & & per square kilometer $\left[\left(\mathrm{ft}^{3} / \mathrm{s}\right) / \mathrm{mi}^{2}\right]$ \\
million gallons per day $(\mathrm{Mgal} / \mathrm{d})$ & 0.04381 & cubic meters per second $\left(\mathrm{m}^{3} / \mathrm{s}\right)$ \\
\hline
\end{tabular}

Temperature in degrees Celsius $\left({ }^{\circ} \mathrm{C}\right)$ can be converted to degrees Fahrenheit $\left({ }^{\circ} \mathrm{F}\right)$ as follows:

$$
{ }^{\circ} \mathrm{F}=1.8\left({ }^{\circ} \mathrm{C}\right)+32
$$

Transmissivity is reported in the standard unit cubic foot per day per square foot times foot of aquifer thickness $\left[\left(\mathrm{ft}^{3} / \mathrm{d}\right) / \mathrm{ft}^{2}\right] \mathrm{ft}$. For convenience, the mathematically reduced form $\mathrm{ft}^{2} / \mathrm{d}$ is used in this report. 


\title{
Relations of Borehole Resistivity to the Horizontal Hydraulic Conductivity and Dissolved-Solids Concentration in Water of Clastic Coastal Plain Aquifers in the Southeastern United States
}

\author{
By Robert E. Faye and Winston G. Smith
}

\begin{abstract}
Aquifer bulk resistivity and grain-surface resistivity (inverse of grain-surface conductance) were tested as geoelectrical analogs to the horizontal hydraulic conductivity of clastic, freshwater aquifers in the Southeastern United States. Bulk resistivity was also tested as a geoelectrical analog for dissolved-solids concentrations in aquifer water. Bulk resistivity was defined as the average resistivity across a contributing interval measured by the long-normal (64-inch) or induction log. Grain-surface resistivity was empirically defined as the difference between aquifer bulk resistivity and aquifer water resistivity (computed from specific conductance). Sources of data were borehole geophysical logs and results of water-quality and aquifer-test analyses related to unconsolidated sands and clayey sands at more than a hundred sites in seven Southeastern States. Waterbearing units were composed of sediments ranging from the Late Cretaceous to middle Eocene.

All bivariate data were related using the logarithmic regression model $Y=A X^{B}$. Aquifer bulk resistivity and grain-surface resistivity were moderately correlated to horizontal hydraulic conductivity (70 and 72 percent correlation coefficients, respectively). Apparent formation factor, defined as the ratio of aquifer bulk resistivity to aquifer water resistivity, was shown to be poorly correlated with horizontal hydraulic conductivity (38 percent correlation
\end{abstract}

coefficient). Aquifer bulk resistivity was shown to be highly correlated with dissolved-solids concentration and aquifer water resistivity (88 and 93 percent correlation coefficients, respectively).

Regression models using bulk resistivity and aquifer water resistivity as independent variables were applied at four locations in South Carolina and Louisiana to predict dissolvedsolids concentrations in aquifer water. Absolute mean error of prediction was 20 and 6 percent, respectively. A regression model using bulk resistivity to predict horizontal hydraulic conductivity was applied at 27 sites in 6 Southeastern States, resulting in an absolute error ranging from 4 to 95 percent with a corresponding mean error of 43 percent.

\section{INTRODUCTION}

This report describes the development and application of several regression models that relate borehole geoelectrical properties to horizontal hydraulic conductivity and the dissolved-solids concentration in water of clastic, unconsolidated aquifers in the Southeastern United States. The regression models are applicable to regional as well as to local aquifer systems composed of unconsolidated sands and clayey sands. The area of investigation extends from the Coastal Plain of North Carolina, across the Atlantic and Gulf Coastal Plains of South Carolina, Georgia, Alabama, Mississippi, and Loui- 
siana, and north into the Mississippi embayment to the vicinity of Memphis, Tenn. (fig. 1, table 1).

Borehole and well data were obtained from more than a hundred sites related to several Coastal Plain rock-stratigraphic units (tables 1,2 ). To simplify the designation of aquifers for a regional investigation, a nomenclature based on chronostratigraphy was used. Aquifers were grouped into three generalized categories: Late Cretaceous, Paleocene and early Eocene, and middle Eocene (table 2). Where the Meridian Sand Formation of middle Eocene age is used for water supply, it generally is developed in combination with sands of the upper Wilcox Group. Accordingly, for this study, data related to the Meridian Sand were grouped with aquifers of Paleocene and early Eocene age.

The general lithology of Coastal Plain clastic aquifers does not vary substantially throughout the study area and is characterized by unconsolidated sand and clayey sand commonly thinly interbedded with clay. Quartz sand is ubiquitous to aquifers of the study area and ranges in size from coarse- to fine-grained. Clay is seldom entirely absent from water-bearing sands and commonly constitutes a substantial part of the aquifer matrix. Much of this clay is probably authigenic (Lee and Strickland, 1988; Chapelle and McMahon, 1991; McMahon and Chapelle, 1991) and occurs within the intergranular spaces of the aquifer matrix. The thickness of sand units in which wells are commonly screened is rarely less than $10 \mathrm{ft}$ and seldom exceeds $200 \mathrm{ft}$ (table 1). Regionally, the thickness of discrete water-bearing sand units decreases and the clay content increases downgradient from outcrops. The dissolved-solids concentration of aquifer waters also generally increases seaward or downgradient from outcrops (Clarke and others, 1983, 1985; Lee, 1988; Pettijohn, 1988).

Hydraulic and water-quality characteristics are highly variable throughout the study area. The dissolved-solids concentration in water samples collected from freshwater aquifers ranges from about 20 to 2,000 milligrams per liter $(\mathrm{mg} / \mathrm{L})$ (tables 3 , 6 ). The observed horizontal hydraulic conductivity $\left(K_{h}\right)$ of Coastal Plain aquifers ranges from about 4 to greater than 500 feet per day (ft/d) (tables 4, 7). Porosity of water-bearing sands regionally varies between 20 and 40 percent but variations within aquifers at a single well or locally between wells appear to be minor (Jones and Buford, 1951; Hosman and others, 1968; Zack, 1977; Cahill, 1982;
Clarke and others, 1985; and Faye and McFadden, 1986). The observed temperature of aquifer water at the point of well discharge ranges from about 16 to $43^{\circ} \mathrm{C}\left(61\right.$ to $110^{\circ} \mathrm{F}$ ) (table 3 ).

Borehole resistivity across intervals of waterbearing sands is highly variable locally (fig. 2) and areally (tables 3,4$)$. Average bulk resistivity $\left(R_{o}\right)$ across contributing water-bearing intervals varies from less than 10 to about $600 \mathrm{ohm}$-meters (ohm-m) throughout the study area. Bulk resistivity represents the total electrical resistance contributed from all sources (grains, matrix material, and water) within the aquifer.

Most transmissivity data (table 4) were obtained from the files of the U.S. Geological Survey. Some transmissivity data were obtained from reports that summarize the results of aquifer-test analyses. These include, primarily, results described by Zack (1977), Aucott and Newcome (1986), Faye and McFadden (1986), and Slack and Darden (1991). Borehole geophysical logs, water-quality data, and well-construction data were, for the most part, obtained from the files of the U.S. Geological Survey (tables 1, 3).

\section{THEORY AND CONCEPTS}

In a clastic, porous media saturated with freshwater (dissolved-solids concentration less than $5,000 \mathrm{mg} / \mathrm{L}$ ), both fluid flow and electrical current move in a tortuous path through the interstices of the media. Accordingly, electrical conductance of the clastic porous media may be substantially influenced by those properties of the media that enhance the ionic content and volume of interstitial water. Such properties can be characterized or measured by determining the specific conductance and dissolvedsolids concentrations of the water, the degree of mineralization of the porous media (particularly the percentage of clay), the particle-size distribution of the clay, silt, and sand grain solids, and the media porosity. Other media properties that probably minimally affect electrical conductance are the sand grain shape and the nature of sand grain packing. Media hydraulic characteristics also vary according to changes in many of these same properties.

Consider that the bulk resistivity of a freshwater aquifer is, for the most part, a function of electrolyte resistivity $\left(R_{e}\right)$ and matrix solids resistivity $\left(R_{m}\right)$; therefore, 


$$
R_{o}=f\left(R_{e}, R_{m}\right)
$$

Electrolyte resistivity is largely a function of the ionic strength of the aquifer water and ion exchange between the water and the surfaces of clays and other fine-grained aquifer solids. This latter process is termed surface conductance (Alger, 1966; Pfannkuch, 1969), the resistance equivalent for which is designated grain-surface resistivity $\left(R_{s}\right)$ in this report; accordingly,

$$
R_{e}=f\left(R_{w}, R_{s}\right)
$$

where $R_{w}$ is the resistivity of aquifer water. The magnitude of grain-surface resistivity decreases with increasing ionic exchange capacity between the aquifer water and the fine-grained aquifer solids exposed to this water (Alger, 1966). Thus, the greater the percentage of clays and sands of small grain size that comprise the aquifer and the greater the ionic content of the aquifer water, the smaller is the related grain-surface resistivity.

Grain-surface resistivity cannot be directly measured. In addition, the geophysical and electrochemical relations of grain-surface resistivity to other borehole geophysical and hydraulic properties are poorly understood and only empirical relations have been developed (Pfannkuch, 1969; Worthington, 1976, 1977; Urish, 1981; Huntley, 1986). A consistent, measurable relation that includes the bulk resistivity and water resistivity components of equations 1 and 2 has been observed, however, such that bulk resistivity always exceeds water resistivity for a saturated porous media (Keys and MacCary, 1971; Engineering Enterprises, Inc., written commun., August 23-24, 1983). Data reported for this study (tables 3, 4) and by Jones and Buford (1951), Turcan (1962), and Brown (1971) indicate that bulk resistivity in Southeastern Coastal Plain clastic, freshwater aquifers is consistently greater than aquifer water resistivity, and that water resistivity comprises a major percentage of bulk resistivity for any contributing interval.

Matrix solids resistivity is the result of electron conductance through the grain-to-grain contacts of contiguous sand grains of the aquifer. Quartz sand is virtually a nonconducting material and matrix solids resistivity is considered infinitely large.

Dimensional analyses by Muskat (1937) and De Wiest (1965) determined that horizontal hydraulic conductivity is directly proportional to the square of the diameter of aquifer pore openings. Therefore, increasing percentages of clay, silt, and fine-grained sand in a clastic aquifer tend to decrease both the average pore diameter and the average horizontal hydraulic conductivity. Such increases in clay and fine-grained sand in freshwater-saturated aquifers also tend to decrease grain-surface resistivity. Accordingly, grain-surface resistivity might be an appropriate geoelectrical analog for the horizontal hydraulic conductivity $\left(K_{h}\right)$ of unconsolidated, clastic porous media; that is

$$
K_{h}=f\left(R_{s}\right) .
$$

Alternatively, the variation of bulk resistivity across water-bearing sands and clayey sands (fig. 2) largely could be the result of changes in clay content, manifest as variations in grain-surface resistivity. Thus, bulk resistivity could also be a suitable geoelectrical analog for horizontal hydraulic conductivity.

To investigate the relations of aquifer bulk resistivity and grain-surface resistivity to horizontal hydraulic conductivity, grain-surface resistivity must first be defined in terms of geoelectrical properties that can be measured in the field. A parallel resistance model, similar to that used by Pfannkuch (1969), is used to relate aquifer bulk resistivity to electrolyte resistivity and matrix solids resistivity,

$$
\frac{1}{R_{o}}=\frac{1}{R_{e}}+\frac{1}{R_{m}}
$$

Because matrix solids resistivity is considered to be infinite, equation 4 reduces to

$$
\begin{gathered}
\frac{1}{R_{o}}=\frac{1}{R_{e}}, \text { and } \\
R_{o}=R_{e}=f\left(R_{w}, R_{s}\right) .
\end{gathered}
$$

Because the exact, deterministic form of equation 2 is unknown, elementary hypothetical relations are used and substituted into equation 6 to evaluate grain-surface resistivity in terms of bulk resistivity and aquifer water resistivity. The hypothetical models applied in this study are a summation model $\left(R_{e}=R_{w}+R_{s}\right)$ and a product model $\left(R_{e}=R_{w} \times R_{s}\right)$.

\section{Summation Model}

The summation model explains grain-surface resistivity as the difference between bulk resistivity 


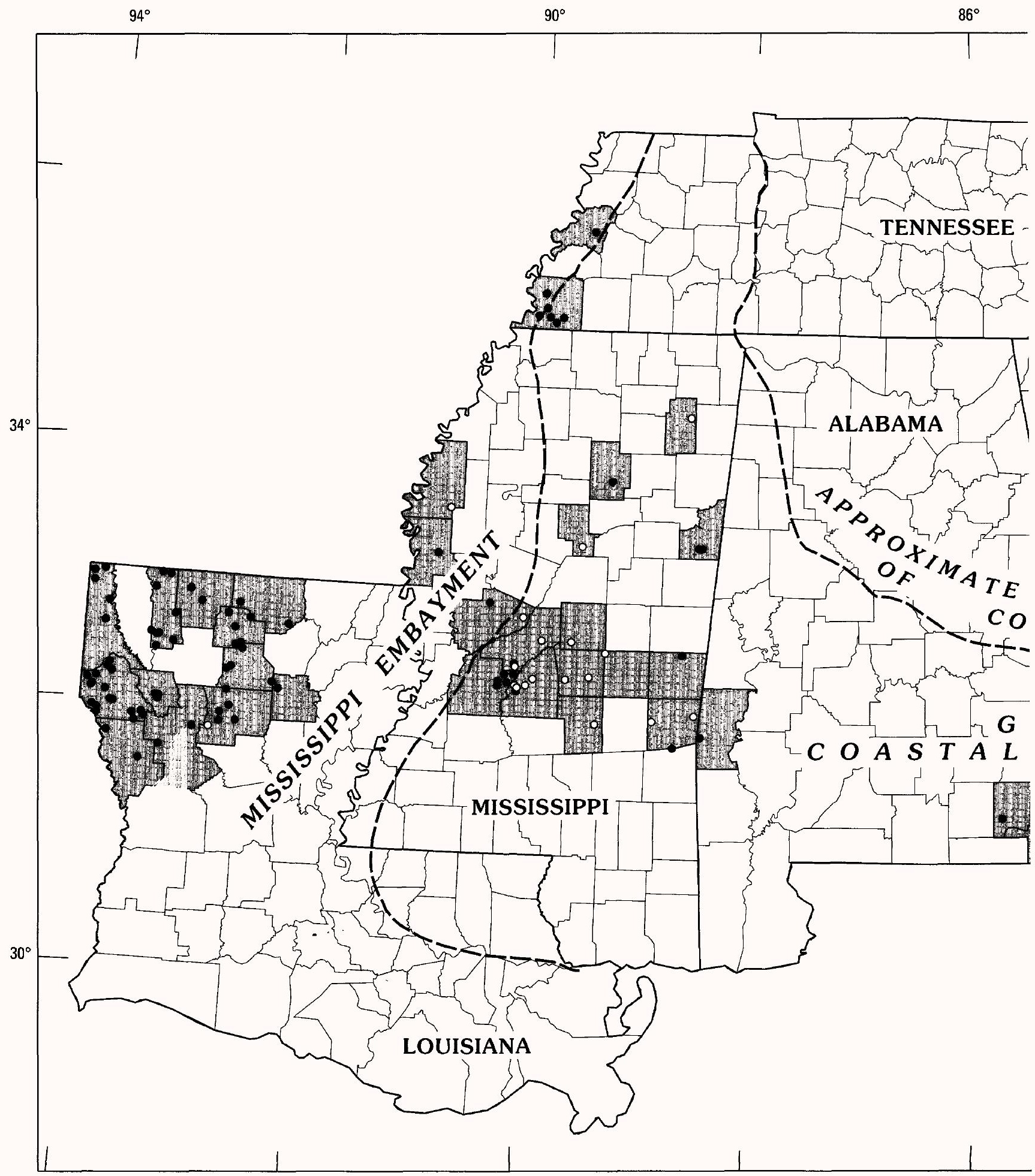

Base modified from U.S. Geological Survey, National Atlas digital data, 1:2,000,000, 1970 Albers Equal-Area Conic projection

Standard parallels $29^{\circ} 30^{\prime}$ and $45^{\circ} 30^{\prime}$, central meridian $-96^{\circ} 00^{\prime}$

Figure 1. Study area and county and site locations of wells. 


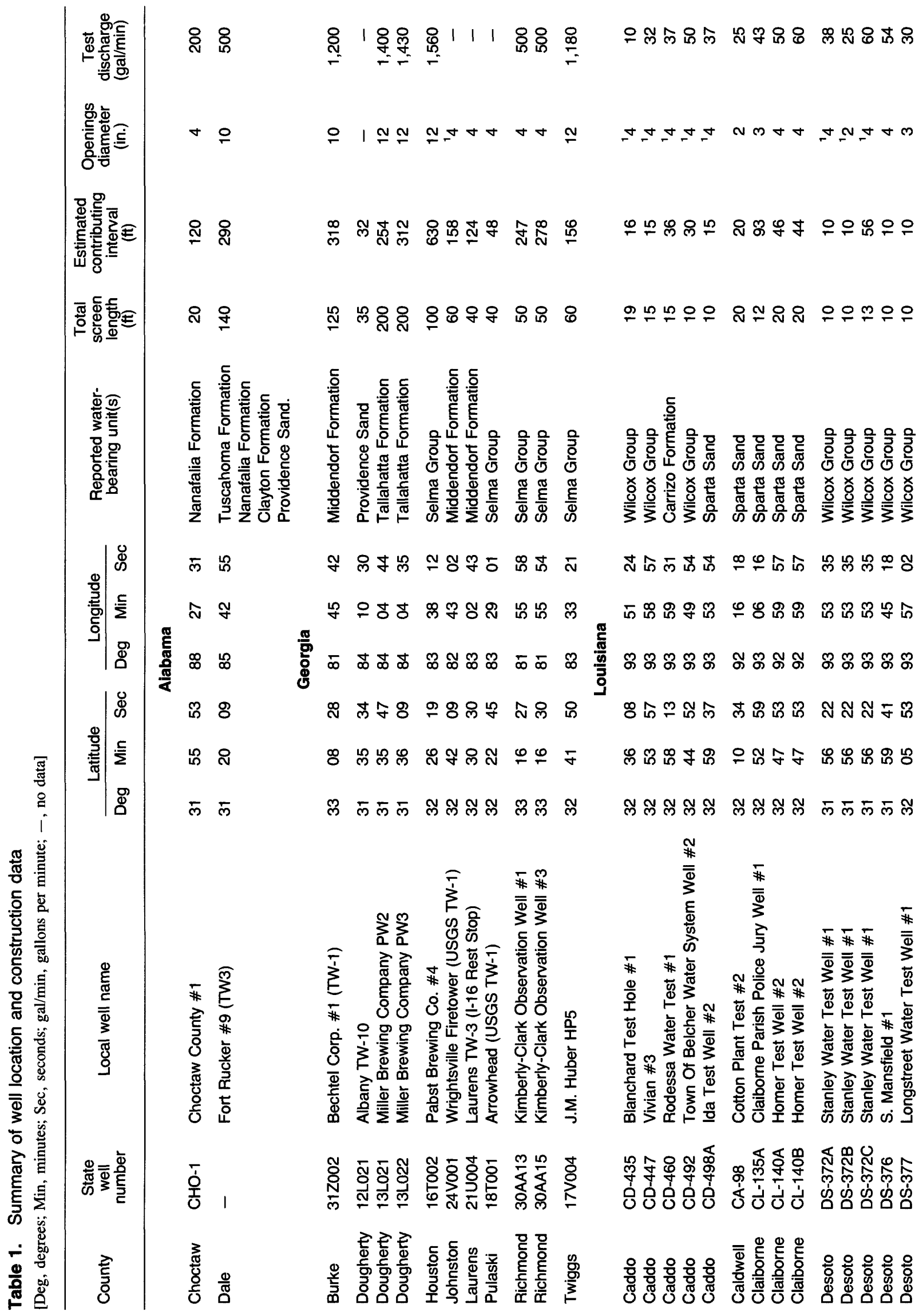




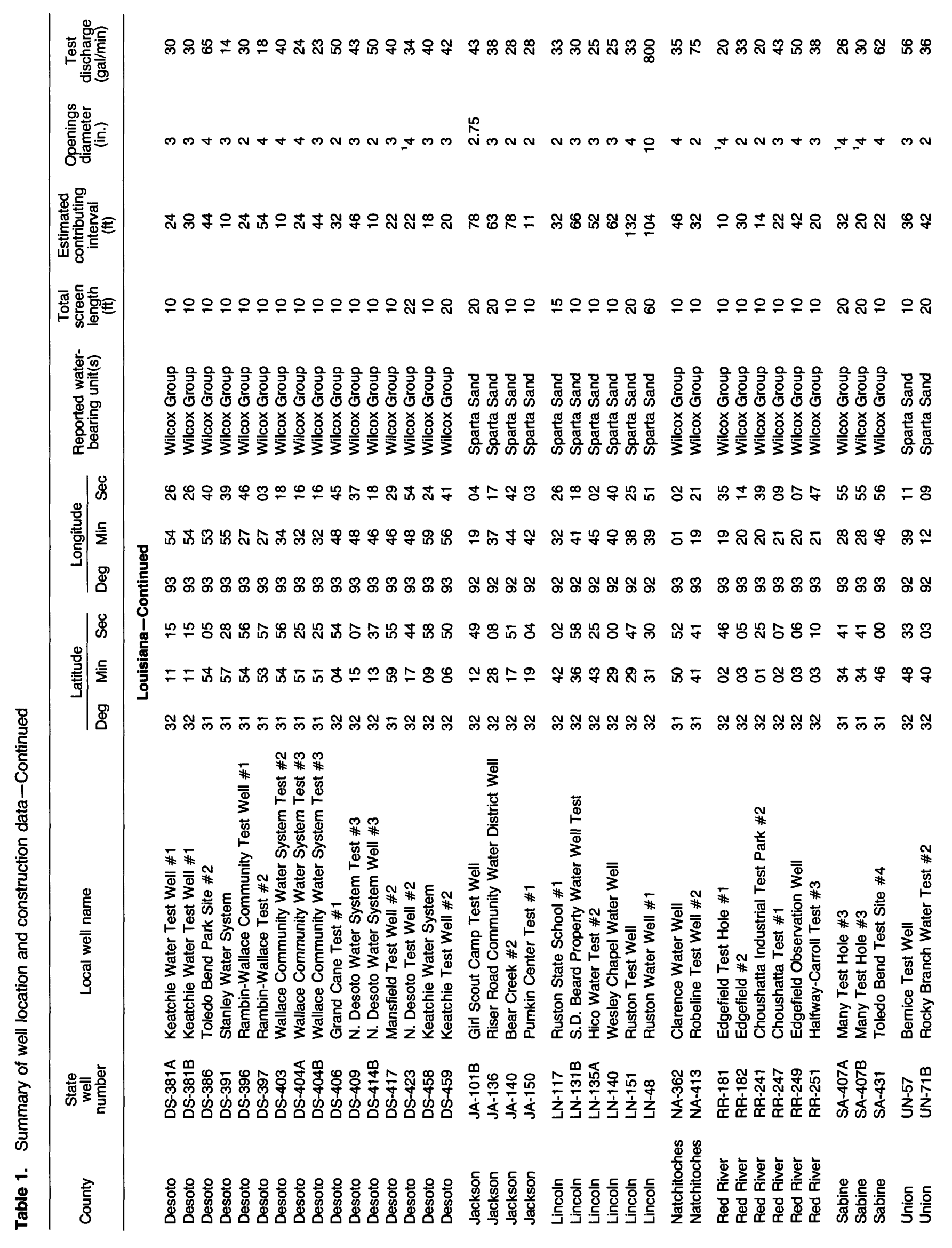




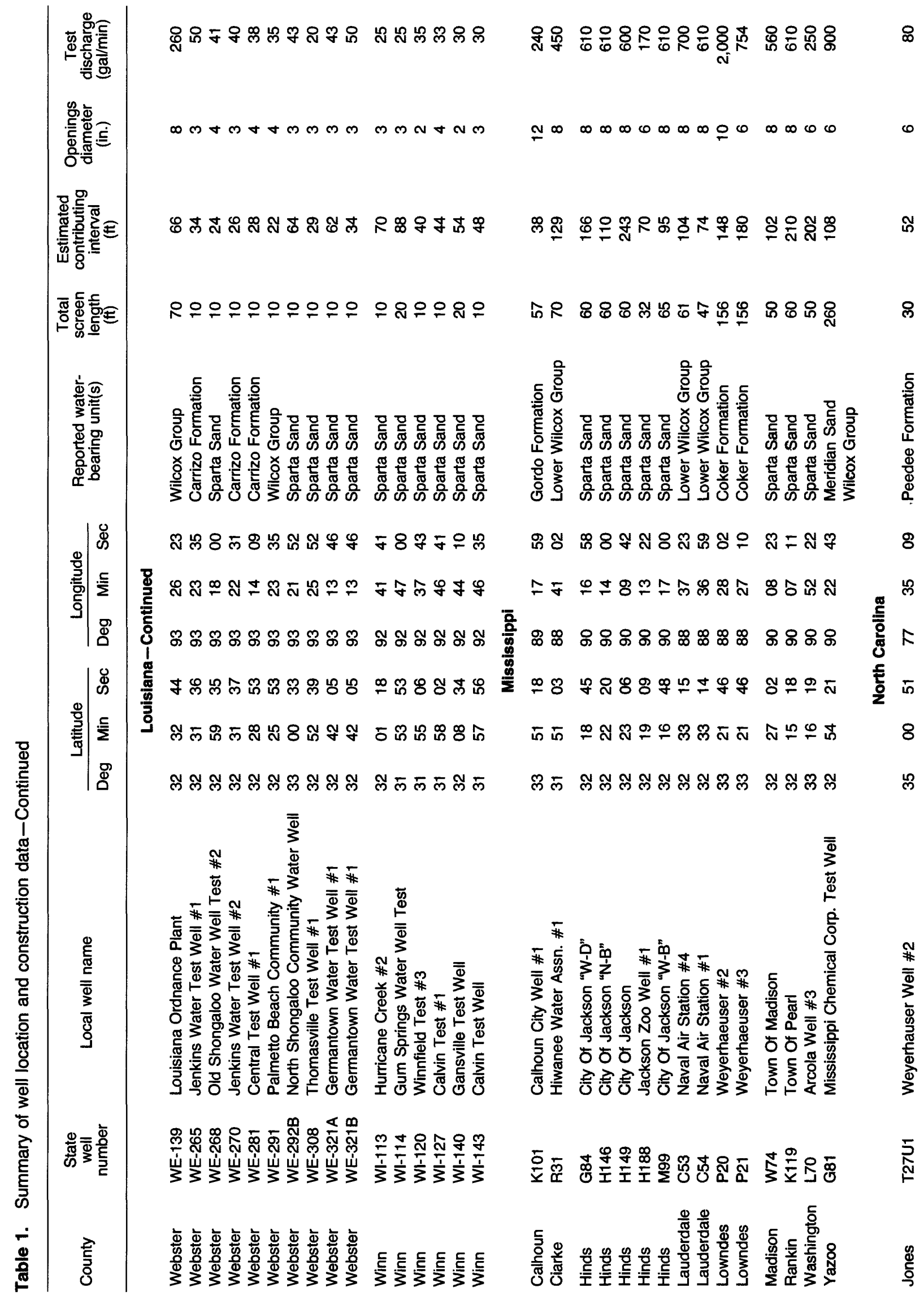




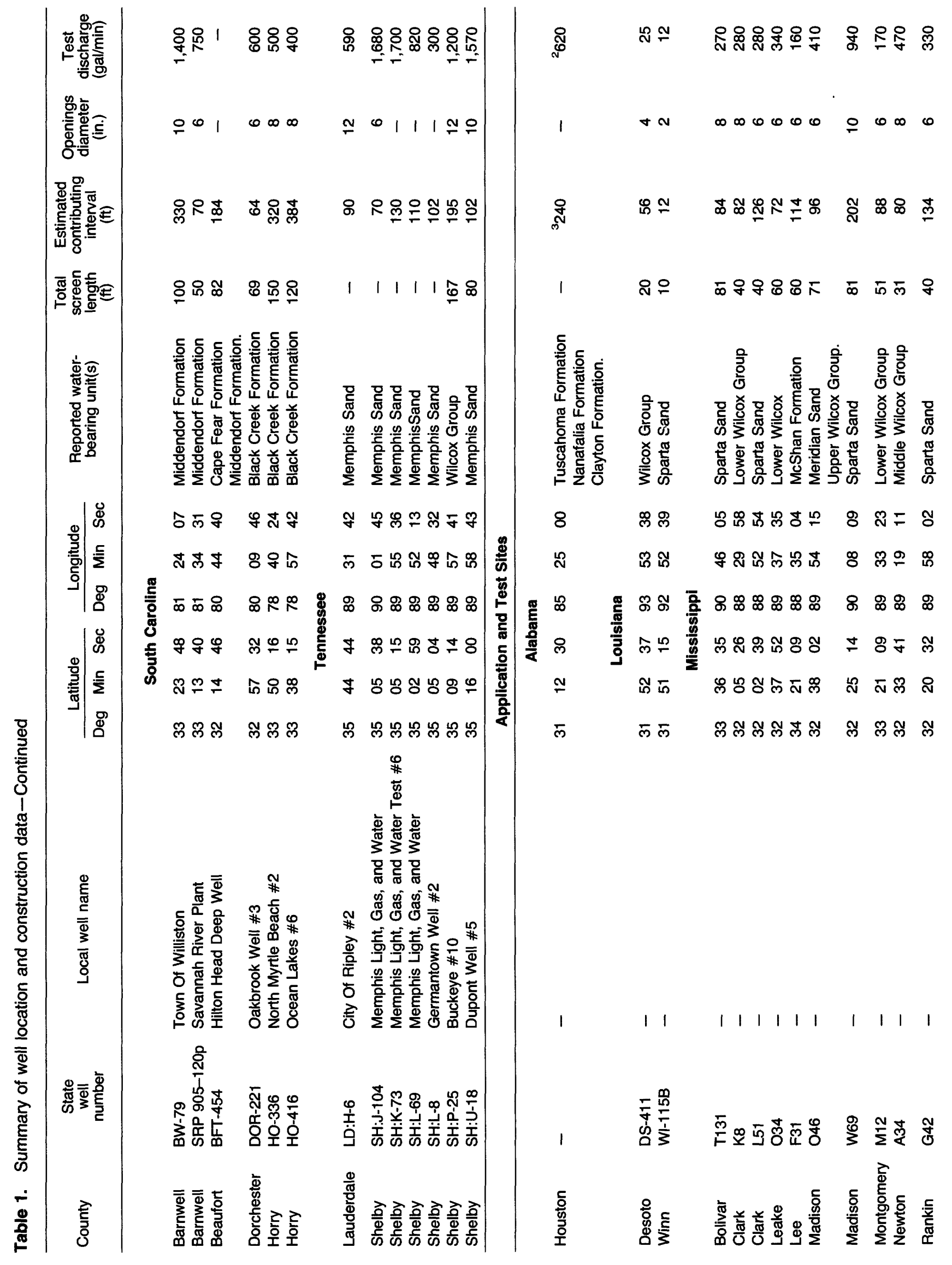




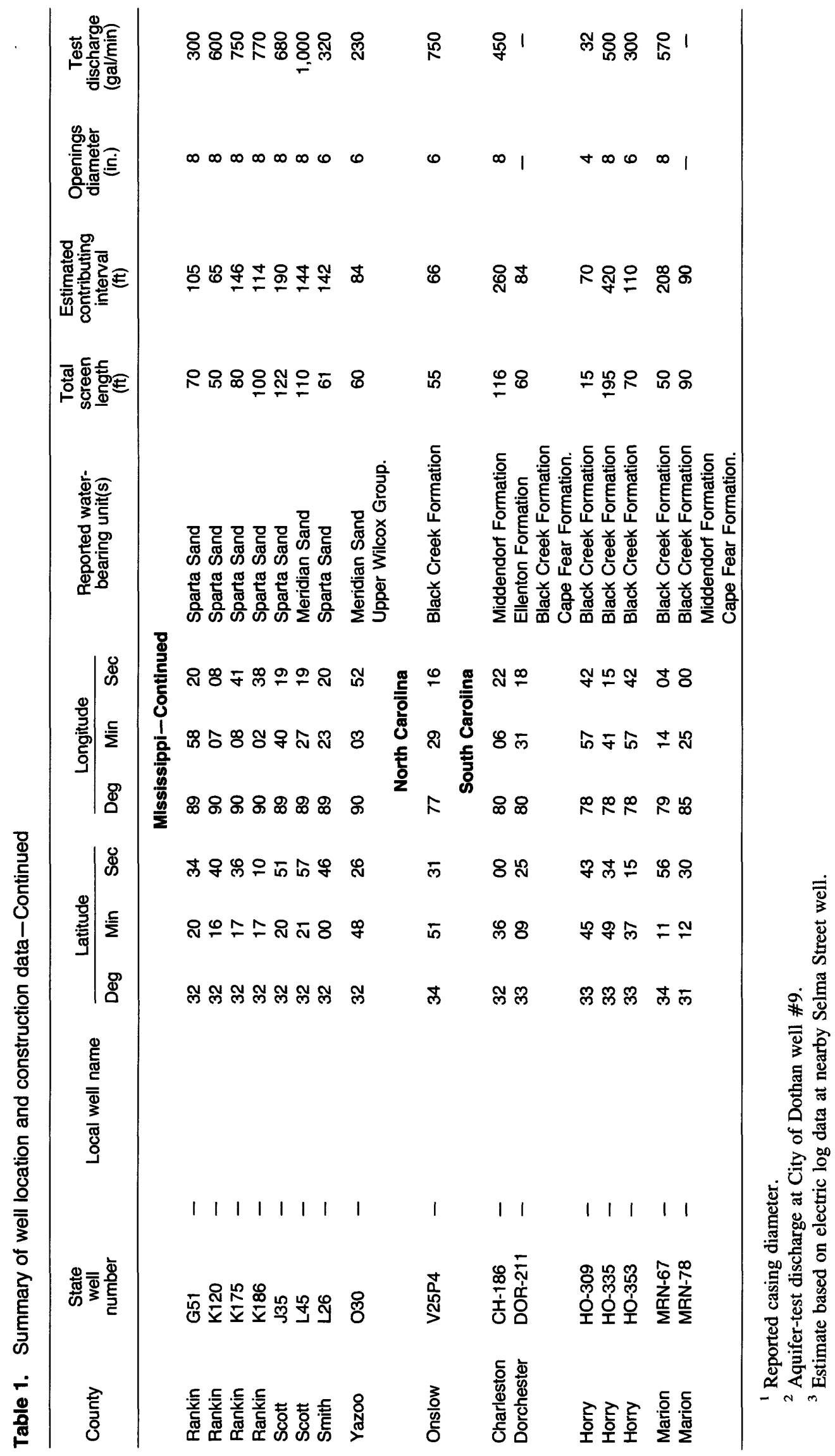


Table 2. Generalized correlation of geologic units and aquifer units

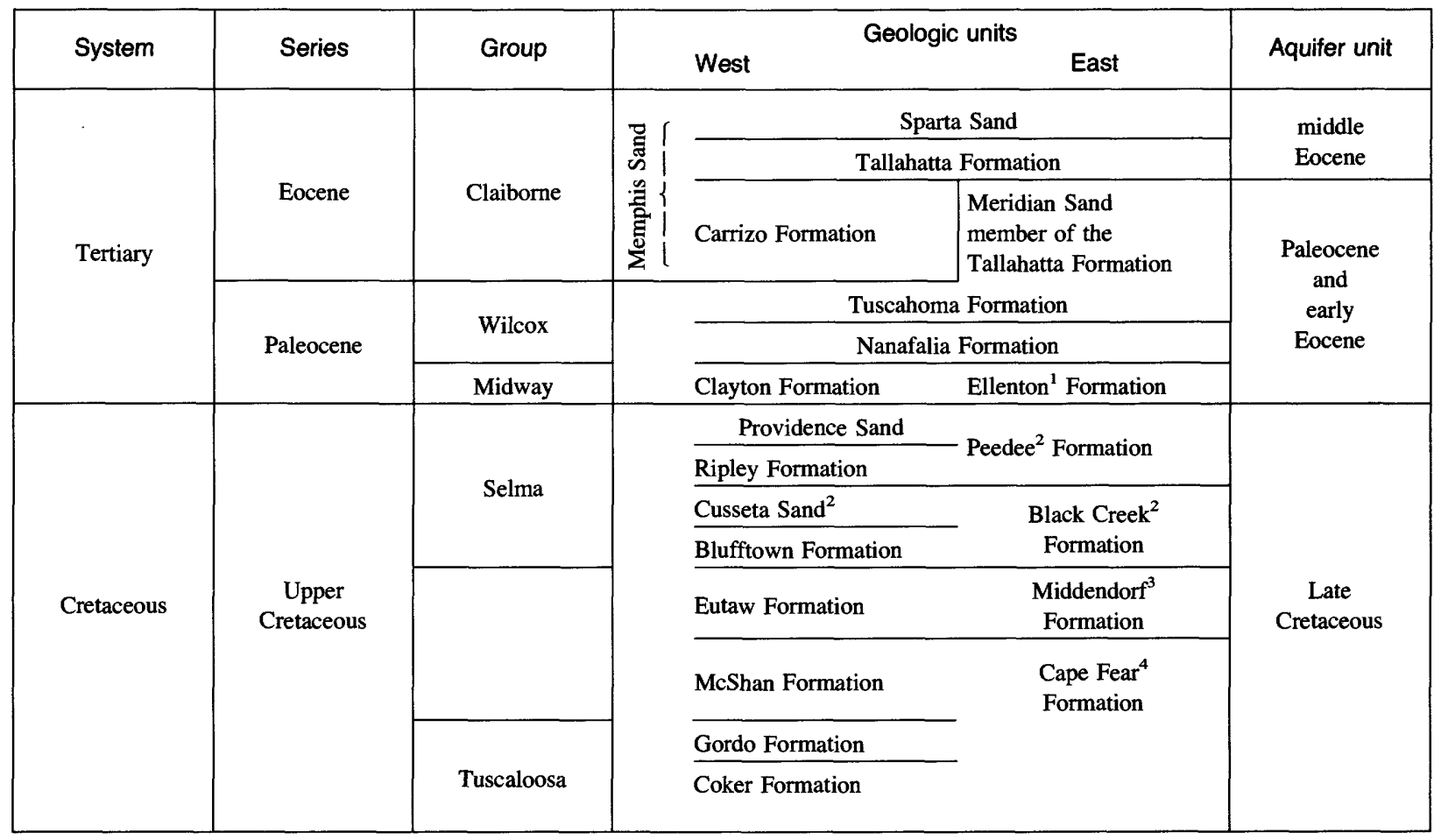

\footnotetext{
${ }^{1}$ Not part of Midway Group.

${ }^{2}$ Not part of Selma Group.

${ }^{3}$ Part of Lumbee Group.

${ }^{4}$ Not part of Tuscaloosa Group.
}

and aquifer water resistivity. Because horizontal hydraulic conductivity is also explained as a function of grain-surface resistivity,

$$
K_{h}=f\left(R_{s}\right)=f\left(R_{o}-R_{w}\right) .
$$

\section{Product Model}

The relation of grain-surface resistivity to bulk resistivity and water resistivity explained by the product model is expressed by the following equation,

$$
K_{h}=\left(f\left(R_{s}\right)=f\left(R_{o} / R_{w}\right) .\right.
$$

The right-hand side of equation 8 generally corresponds to the definition of apparent formation factor $\left(F_{a}\right)$ described by Pfannkuch (1969) and Worthington (1977). Apparent formation factor is the ratio of bulk resistivity to water resistivity in freshwater- saturated porous media containing clays or other conductive solids and previously has been used to estimate horizontal hydraulic conductivity (Alger, 1966; Croft, 1971; Urish, 1981; Biella and others, 1983; Alger and Harrison, 1989). Intrinsic formation factor $(F)$ is similarly defined but applies only to porous media saturated with highly conductive electrolytes, such as brines (Archie, 1942). Where porous media are saturated with brine, geoelectrical conductance is almost entirely through the electrolyte (pore spaces); grain-surface conductance is minimal. All subsequent references to formation factor are to apparent formation factor.

Application of equations 7 and 8 at well sites requires a data base that includes aquifer-test results or laboratory determinations of hydraulic characteristics, borehole lithologic and well construction logs, calibrated borehole resistivity logs, and chemical analyses of water samples from the completed wells. 
Table 3. Summary of borehole geoelectrical properties and aquifer water-quality characteristics at well sites [ohm-m, ohm-meter; $\mu \mathrm{S} / \mathrm{cm}$, microsiemens per centimeter; $\mathrm{mg} / \mathrm{L}$, milligrams per liter; - , no data]

\begin{tabular}{|c|c|c|c|c|c|c|c|c|}
\hline County & $\begin{array}{l}\text { State well } \\
\text { number }\end{array}$ & Local well name & $\begin{array}{l}\text { Average } \\
\text { bulk } \\
\text { resistivity } \\
\left(R_{0}\right) \\
\text { (ohm-m) }\end{array}$ & $\begin{array}{c}\text { Water } \\
\text { discharge } \\
\text { temperature } \\
\left({ }^{\circ} \mathrm{C}\right)\end{array}$ & $\begin{array}{c}\text { Specific } \\
\text { conductance of } \\
\text { well discharge } \\
(\mu \mathrm{S} / \mathrm{cm})\end{array}$ & $\begin{array}{c}\text { Water } \\
\text { resistivity } \\
\text { at } 25^{\circ} \mathrm{C}\left(R_{w}\right) \\
(\text { ohm-m) }\end{array}$ & $\begin{array}{l}\text { Water resistivity } \\
\text { at discharge } \\
\text { temperature }\left(R_{w x}\right) \\
\text { (ohm- } \mathrm{m})\end{array}$ & $\begin{array}{c}\text { Dissolved-solids } \\
\text { concentration of } \\
\text { well discharge } \\
(\mathrm{mg} / \mathrm{L})\end{array}$ \\
\hline \multicolumn{9}{|c|}{ Alabama } \\
\hline Choctaw & CHO-1 & Choctaw County \#1 & 31 & 33.0 & 900 & 11.1 & 9.5 & ${ }^{1} 535$ \\
\hline Dale & - & Fort Rucker \#9 (TW-3) & 120 & 23.5 & 320 & 31.3 & 32.3 & - \\
\hline \multicolumn{9}{|c|}{ Georgia } \\
\hline Burke & $31 Z 002$ & Bechtel Corp. \#1 (TW-1) & 200 & - & ${ }^{2} 180$ & 55.6 & 55.6 & - \\
\hline Dougherty & $12 \mathrm{~L} 021$ & Albany TW-10 & 30 & 24.0 & 560 & 17.9 & 18.3 & ${ }^{3} 242$ \\
\hline Dougherty & $13 \mathrm{~L} 021$ & Miller Brewing Company PW2 & 100 & 20.0 & 321 & 31.2 & 34.9 & 192 \\
\hline Dougherty & $13 \mathrm{~L} 022$ & Miller Brewing Company PW3 & 66 & 20.0 & 321 & 31.2 & 34.9 & 192 \\
\hline Houston & 16T002 & Pabst Brewing Co. \#4 & 580 & 19.0 & 35 & 286 & 328 & 431 \\
\hline Johnston & 24V001 & $\begin{array}{l}\text { Wrightsville Firetower (USGS } \\
\text { TW-1). }\end{array}$ & 110 & 30.0 & 145 & 69 & 62.3 & ${ }^{4} 112$ \\
\hline Laurens & 21U004 & Laurens TW-3 (I-16 Rest Stop) & 120 & 24.9 & 115 & 87 & 87.2 & ${ }^{4} 75$ \\
\hline Pulaski & $18 \mathrm{~T} 001$ & Arrowhead (USGS TW-1) & 280 & 24.5 & 79 & 127 & 128 & ${ }^{4} 26$ \\
\hline Richmond & $30 \mathrm{AA} 13$ & $\begin{array}{l}\text { Kimberly-Clark Observation } \\
\text { Well \#1. }\end{array}$ & 170 & 25.0 & 120 & 83.3 & 83.3 & 88 \\
\hline Richmond & 30AA15 & $\begin{array}{l}\text { Kimberly-Clark Observation } \\
\text { Well \#3. }\end{array}$ & 200 & 25.0 & 107 & 93.5 & 93.5 & 91 \\
\hline Twiggs & $17 \mathrm{~V} 004$ & J.M. Huber HP5 & 430 & - & 46 & 217 & 217 & $5_{47}$ \\
\hline \multicolumn{9}{|c|}{ Louisiana } \\
\hline Caddo & $\mathrm{CD}-435$ & Blanchard Test Hole \#1 & 21 & 20.6 & 916 & 10.9 & 12.0 & 517 \\
\hline Caddo & $\mathrm{CD}-447$ & Vivian \#3. & 32 & - & 697 & 14.3 & 14.3 & 419 \\
\hline Caddo & $\mathrm{CD}-460$ & Rodessa Water Test \# 1 & 33 & - & 916 & 10.9 & 10.9 & 204 \\
\hline Caddo & $\mathrm{CD}-492$ & $\begin{array}{l}\text { Town Of Belcher Water System } \\
\text { Well \#2. }\end{array}$ & 15 & - & 1,550 & 6.5 & 6.5 & 902 \\
\hline Caddo & $\mathrm{CD}-498 \mathrm{~A}$ & Ida Test Well \#2 & 45 & - & 273 & 36.6 & 36.6 & 151 \\
\hline Caldwell & CA-98 & Cotton Plant Test \#2 & 18 & - & 1,360 & 7.4 & 7.4 & 836 \\
\hline Claiborne & CL-135A & $\begin{array}{l}\text { Claiborne Parish Police Jury } \\
\text { Well \#1. }\end{array}$ & 160 & - & 139 & 71.9 & 71.9 & 170 \\
\hline Claiborne & CL-140A & Homer Test Well \#2 & 140 & - & 190 & 52.6 & 52.6 & 137 \\
\hline Claiborne & CL-140B & Homer Test Well \#2 & 150 & - & 200 & 50.0 & 50.0 & 169 \\
\hline Desoto & DS-372A & Stanley Water Test Well \#1 & 100 & - & 189 & 52.9 & 52.9 & 162 \\
\hline Desoto & DS-372B & Stanley Water Test Well \#1 & 23 & 21.6 & 1,160 & 8.6 & 9.3 & 738 \\
\hline Desoto & DS-372C & Stanley Water Test Well \# 1 & 29 & 21.6 & 1,310 & 7.6 & 8.2 & 805 \\
\hline Desoto & DS-376 & S. Mansfield \#1 & 33 & 21.7 & 607 & 16.5 & 17.8 & 379 \\
\hline Desoto & DS-377 & Longstreet Water Test Well \#1 & 27 & 21.9 & 772 & 13.0 & 13.9 & 463 \\
\hline Desoto & DS-381A & Keatchie Water Test Well \#1 & 27 & 23.3 & 765 & 13.1 & 13.6 & 458 \\
\hline Desoto & DS-381B & Keatchie Water Test Well \#1 & 30 & 22.8 & 885 & 11.3 & 11.9 & 526 \\
\hline Desoto & DS-386 & Toledo Bend Site \#2 & 27 & 21.1 & 1,510 & 6.6 & 7.2 & 889 \\
\hline Desoto & DS-391 & Stanley Water System & 29 & 21.7 & 1,800 & 5.6 & 6.0 & 1,070 \\
\hline Desoto & DS-396 & $\begin{array}{l}\text { Rambin-Wallace Community Test } \\
\text { Well \#1. }\end{array}$ & 90 & 20.5 & 384 & 26.0 & 28.8 & 260 \\
\hline Desoto & DS-397 & Rambin-Wallace Test \#2 & 30 & 21.5 & 1,030 & 9.7 & 10.5 & 681 \\
\hline Desoto & DS-403 & $\begin{array}{l}\text { Wallace Community Water System } \\
\text { Test \#2. }\end{array}$ & 40 & 21.1 & 403 & 24.8 & 27.1 & 267 \\
\hline Desoto & DS-404A & $\begin{array}{l}\text { Wallace Community Water System } \\
\text { Test \#3. }\end{array}$ & 27 & 21.5 & 772 & 13.0 & 14.1 & 481 \\
\hline Desoto & DS-404B & $\begin{array}{l}\text { Wallace Community Water System } \\
\text { Test \#3. }\end{array}$ & 27 & 22.5 & 768 & 13.0 & 13.7 & 469 \\
\hline Desoto & DS-406 & Grand Cane Test \#1 & 30 & 21.0 & 570 & 17.5 & 19.1 & 341 \\
\hline Desoto & DS-409 & N. Desoto Water System Test \#3 & 27 & 23.9 & 1,620 & 6.2 & 6.3 & 921 \\
\hline Desoto & DS-414B & N. Desoto Water System Well \#3 & 20 & - & 1,660 & 6.0 & 6.0 & 940 \\
\hline
\end{tabular}


Table 3. Summary of borehole geoelectrical properties and aquifer water-quality characteristics at well sites-Continued

\begin{tabular}{|c|c|c|c|c|c|c|c|c|}
\hline County & $\begin{array}{l}\text { State well } \\
\text { number }\end{array}$ & Local well name & $\begin{array}{c}\text { Average } \\
\text { bulk } \\
\text { resistivity } \\
\left(R_{0}\right) \\
\text { (ohm-m) }\end{array}$ & $\begin{array}{c}\text { Water } \\
\text { discharge } \\
\text { temperature } \\
\left({ }^{\circ} \mathrm{C}\right)\end{array}$ & $\begin{array}{c}\text { Specific } \\
\text { conductance of } \\
\text { well discharge } \\
(\mu \mathrm{S} / \mathrm{cm})\end{array}$ & $\begin{array}{c}\text { Water } \\
\text { resistivity } \\
\text { at } 25^{\circ} \mathrm{C}\left(R_{w}\right) \\
(\mathrm{ohm}-\mathrm{m})\end{array}$ & $\begin{array}{l}\text { Water resistivity } \\
\text { at discharge } \\
\text { temperature }\left(R_{w x}\right) \\
\text { (ohm- m) }\end{array}$ & $\begin{array}{l}\text { Dissolved-solids } \\
\text { concentration of } \\
\text { well discharge } \\
\text { (mg/L) }\end{array}$ \\
\hline \multicolumn{9}{|c|}{ Louisiana-Continued } \\
\hline Desoto & DS-417 & Mansfield Test Well \#2 & 27 & - & 953 & 10.5 & 10.5 & 568 \\
\hline Desoto & DS-423 & N. Desoto Test Well \#2 & 40 & 22.2 & 539 & 18.6 & 19.8 & 331 \\
\hline Desoto & DS-459 & Keatchie Test Well \#2 & 24 & 22.0 & 779 & 12.8 & 13.7 & 457 \\
\hline Jackson & JA-101B & Girl Scout Camp Test Well & 37 & 22.5 & 1,090 & 9.2 & 9.7 & 681 \\
\hline Jackson & JA-136 & $\begin{array}{l}\text { Riser Road Community Water } \\
\text { District Well. }\end{array}$ & 45 & 25.6 & 536 & 18.7 & 18.5 & 331 \\
\hline Jackson & JA-140 & Bear Creek \#2 & 120 & 21.9 & 243 & 41.2 & 44.1 & 191 \\
\hline Jackson & JA-150 & Pumkin Center Test \#1 & 30 & 24.4 & 402 & 24.9 & 25.2 & 258 \\
\hline Lincoln & LN-151 & Ruston Test Well & 60 & 26.0 & 408 & 24.5 & 24.0 & 274 \\
\hline Lincoln & LN-48 & Ruston Water Well \#1 & 150 & 25.0 & 252 & 39.7 & 39.7 & 187 \\
\hline Natchitoches & NA-362 & Clarence Water Well & 53 & 20.6 & 636 & 15.7 & 17.3 & 403 \\
\hline Natchitoches & NA-413 & Robeline Test Well \#2 & 25 & 23.1 & 1,240 & 8.1 & 8.4 & 789 \\
\hline Red River & RR-181 & Edgefield Test Hole \#1 & 46 & 25.8 & 345 & 29.0 & 28.5 & 217 \\
\hline Red River & RR-182 & Edgefield \#2 & 88 & - & 308 & 32.5 & 32.5 & 206 \\
\hline Red River & RR-241 & $\begin{array}{l}\text { Choushatta Industrial Park } \\
\text { Test \#2. }\end{array}$ & 26 & 21.1 & 783 & 12.8 & 14.0 & 464 \\
\hline Red River & RR-247 & Choushatta Test \#1 & 32 & 19.4 & 706 & 14.2 & 16.1 & 396 \\
\hline Red River & RR-249 & Edgefield Observation Well & 55 & 18.3 & 265 & 37.7 & 44.0 & 188 \\
\hline Red River & RR-251 & Halfway-Carroll Test \#3 & 22 & 20.0 & 773 & 12.9 & 14.4 & 450 \\
\hline Webster & WE-265 & Jenkins Water Test Well \#1 & 26 & 21.1 & 1,790 & 5.6 & 6.1 & 974 \\
\hline Webster & WE-268 & $\begin{array}{l}\text { Old Shongaloo Water Well } \\
\text { Test \#2. }\end{array}$ & 40 & 22.2 & 472 & 21.2 & 22.6 & 290 \\
\hline Webster & WE-270 & Jenkins Water Test Well \#2 & 34 & 21.7 & 1,060 & 9.4 & 10.1 & 603 \\
\hline Webster & WE-281 & Central Test Well \#1 & 38 & 23.9 & 689 & 14.5 & 14.9 & 434 \\
\hline Webster & WE-291 & Palmetto Beach Community \#1 & 38 & 19.4 & 421 & 23.8 & 27.0 & 265 \\
\hline Webster & WE-292B & $\begin{array}{l}\text { North Shongaloo Community } \\
\text { Water Well. }\end{array}$ & 54 & 20.6 & 611 & 16.4 & 18.1 & 365 \\
\hline Webster & WE-308 & Thomasville Test Well \#1 & 80 & 21.1 & 322 & 31.1 & 33.9 & 200 \\
\hline Webster & WE-321A & Germantown Water Test Well \#1 & 160 & 22.8 & 168 & 59.5 & 62.4 & 150 \\
\hline Webster & WE-321B & Germantown Water Test Well \#1 & 160 & - & 201 & 49.8 & 49.8 & 179 \\
\hline Winn & WI-113 & Hurricane Creek \#2 & 34 & 20.6 & 1,250 & 8.0 & 8.8 & 699 \\
\hline Winn & WI-114 & Gum Springs Water Well Test & 30 & 24.4 & 897 & 11.1 & 11.2 & 501 \\
\hline Winn & WI-120 & Winnfield Test \#3 & 30 & - & 840 & 11.9 & 11.9 & 494 \\
\hline Winn & WI-127 & Calvin Test \#1 & 32 & 21.7 & 979 & 10.2 & 11.0 & 566 \\
\hline Winn & WI-140 & Gansville Test Well & 37 & 22.2 & 851 & 11.8 & 12.6 & 528 \\
\hline Winn & WI-143 & Calvin Test Well & 27 & 22.2 & 1,080 & 9.3 & 9.9 & 603 \\
\hline \multicolumn{9}{|c|}{ Mississippi } \\
\hline Calhoun & K101 & Calhoun City Well \#3 & 41 & 33.3 & 1,135 & 8.8 & 7.5 & - \\
\hline Clarke & R31 & Hiwanee Water Assn. \#1 & 35 & 38.5 & 800 & 12.5 & 9.7 & 605 \\
\hline
\end{tabular}


Table 3. Summary of borehole geoelectrical properties and aquifer water-quality characteristics at well sites-Continued

\begin{tabular}{|c|c|c|c|c|c|c|c|c|}
\hline County & $\begin{array}{l}\text { State well } \\
\text { number }\end{array}$ & Local well name & $\begin{array}{c}\text { Average } \\
\text { bulk } \\
\text { resistivity } \\
\left(R_{o}\right) \\
\text { (ohm-m) }\end{array}$ & $\begin{array}{c}\text { Water } \\
\text { discharge } \\
\text { temperature } \\
\left({ }^{\circ} \mathrm{C}\right)\end{array}$ & $\begin{array}{c}\text { Specific } \\
\text { conductance of } \\
\text { well discharge } \\
(\mu \mathrm{S} / \mathrm{cm})\end{array}$ & $\begin{array}{c}\text { Water } \\
\text { resistivity } \\
\text { at } 25^{\circ} \mathrm{C}\left(R_{w}\right) \\
(\text { ohm-m) }\end{array}$ & $\begin{array}{l}\text { Water resistivity } \\
\text { at discharge } \\
\text { temperature }\left(R_{w x}\right) \\
\text { (ohm- m) }\end{array}$ & $\begin{array}{c}\text { Dissolved-solids } \\
\text { concentration of } \\
\text { well discharge } \\
\text { (mg/L) }\end{array}$ \\
\hline \multicolumn{9}{|c|}{ Mississippi-Continued } \\
\hline Hinds & G84 & City Of Jackson "W-D" & 55 & 29.0 & 420 & 23.8 & 21.9 & 362 \\
\hline Hinds & H146 & City Of Jackson "N-B" & 78 & 28.0 & 390 & 25.6 & 24.1 & 267 \\
\hline Hinds & H188 & Jackson Zoo Well \#1 & 26 & 26.0 & 449 & 22.3 & 21.8 & 275 \\
\hline Hinds & M99 & City Of Jackson "W-B" & 60 & 30.6 & 420 & 23.8 & 21.3 & 337 \\
\hline Lauderdale & C53 & Naval Air Station \#4 & 400 & - & 61 & 164 & 164 & 66 \\
\hline Lauderdale & C54 & Naval Air Station \#1 & 400 & - & 68 & 147 & 147 & 54 \\
\hline Lowndes & $\mathbf{P} 20$ & Weyerhaeuser \#2 & 150 & - & 93 & 107 & 107 & 16 \\
\hline Lowndes & P21 & Weyerhaeuser \#3 & 150 & - & 117 & 85.5 & 85.5 & 22 \\
\hline Madison & W74 & Town Of Madison & 40 & 33.0 & 387 & 25.8 & 22.0 & 231 \\
\hline Rankin & K119 & Town Of Pearl & 40 & 31.0 & 370 & 27.0 & 23.9 & 217 \\
\hline Washington & L70 & Arcola Well \#3 & 40 & 27.0 & 610 & 16.4 & 15.7 & 479 \\
\hline Yazoo & G81 & $\begin{array}{l}\text { Mississippi Chemical Corp. } \\
\text { Test Well. }\end{array}$ & 70 & 35.0 & 670 & 14.9 & 12.3 & 408 \\
\hline \multicolumn{9}{|c|}{ North Carohina } \\
\hline Jones & T27U1 & Weyerhauser Well \#2 & 150 & - & 280 & 35.7 & 35.7 & 184 \\
\hline \multicolumn{9}{|c|}{ South Carolina } \\
\hline Barnwell & BW-79 & Town Of Williston & 270 & 20.0 & 56 & 179 & 200 & 36 \\
\hline Barnwell & SRP905-120P & Savannah River Plant & 610 & - & 46 & 217 & 217 & 38 \\
\hline Beaufort & BFT-454 & Hilton Head Deep Well & 6.2 & 43.5 & 1,900 & 5.3 & 3.8 & 1,310 \\
\hline Dorchester & DOR-221 & Oakbrook Well \#3 & 11 & 31.1 & 980 & 10.2 & 9.0 & - \\
\hline Horry & HO-336 & North Myrtle Beach \#2 & 7.8 & 26.5 & 1,850 & 5.4 & 5.2 & 1,150 \\
\hline Horry & HO-416 & Ocean Lakes \#6 & 17 & 24.0 & 1,080 & 9.3 & 9.5 & 670 \\
\hline \multicolumn{9}{|c|}{ Tennessee } \\
\hline Lauderdale & LD:H-6 & City Of Ripley \#2 & 200 & 18.3 & 187 & 53.5 & 62.5 & 104 \\
\hline Shelby & SH:J-104 & $\begin{array}{l}\text { Memphis Light, Gas, and } \\
\text { Water. }\end{array}$ & 300 & 17.2 & 143 & 69.9 & 83.9 & 80 \\
\hline Shelby & SH:K-73 & $\begin{array}{l}\text { Memphis Light, Gas, and } \\
\text { Water Test \#6. }\end{array}$ & 380 & 16.1 & 148 & 67.6 & 83.5 & 89 \\
\hline Shelby & SH:L-69 & $\begin{array}{l}\text { Memphis Light, Gas, and } \\
\text { Water. }\end{array}$ & 400 & 16.7 & 88 & 114 & 138 & 54 \\
\hline Shelby & SH:L-8 & Germantown Well \#2 & 550 & 17.2 & 63 & 159 & 190 & 47 \\
\hline Shelby & SH:P-25 & Buckeye \#10 & 190 & 21.7 & 174 & 57.5 & 61.9 & 125 \\
\hline Shelby & SH:U-18 & Dupont Well \#5 & 160 & 19.4 & 180 & 55.6 & 63.2 & 180 \\
\hline
\end{tabular}

${ }^{1}$ Davis and others (1983).

${ }^{2}$ Water-quality data from makeup well \#2 (K.R. Davis, Georgia Geologic Survey, written commun., 1989).

${ }^{3}$ Lee (1984).

${ }^{4}$ Water-quality data from Clarke and others, 1985.

${ }^{5}$ Water-quality data from well HP-6 (K.R. Davis, Georgia Geologic Survey, written commun., October 4, 1989). 


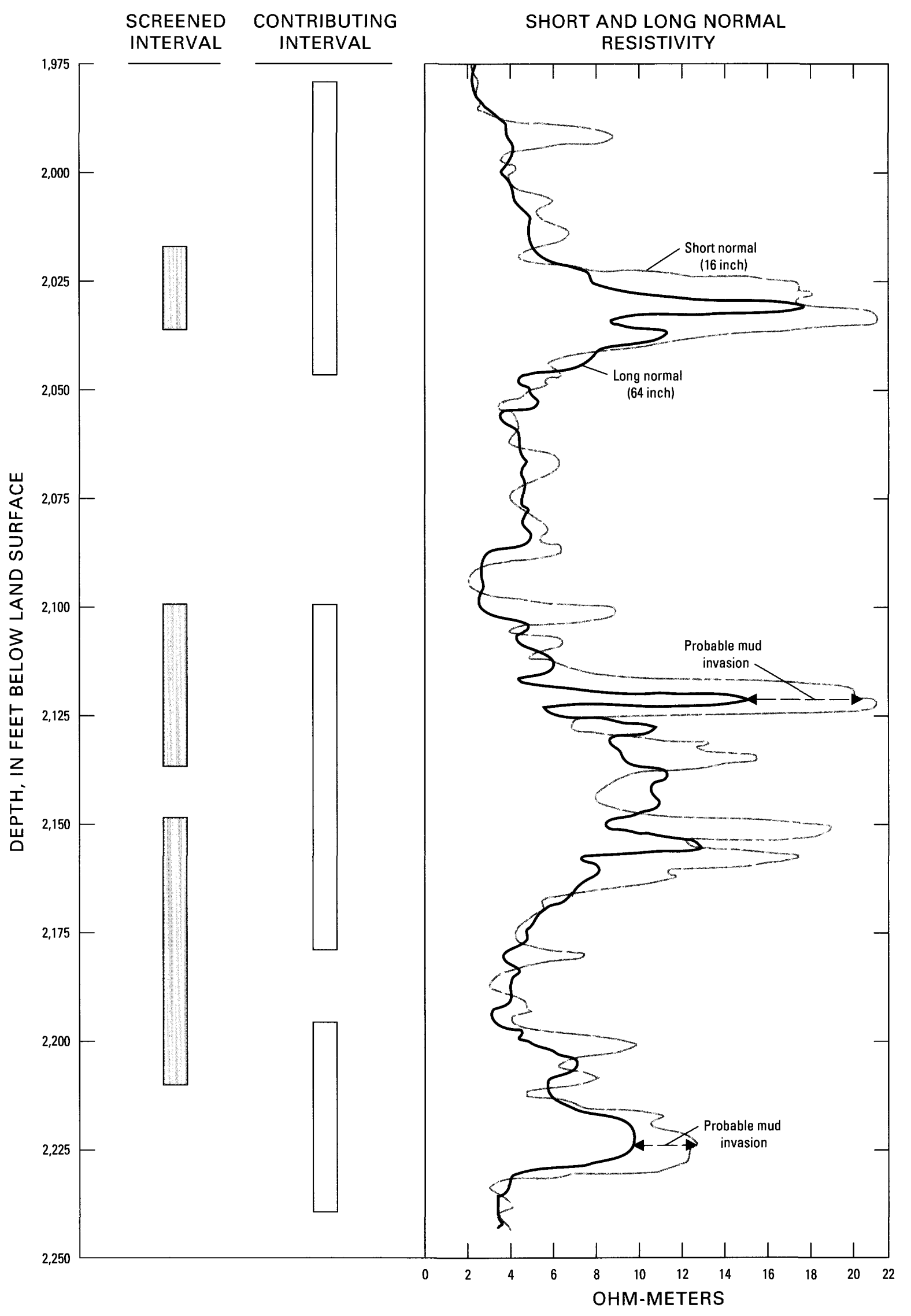

Figure 2. Resistivity borehole log at site $\mathrm{CH}-186$ showing short- and long-normal curves and the screened and estimated contributing intervals. 
Table 4. Summary of borehole geoelectrical properties and aquifer hydraulic characteristics at well sites-Continued

\begin{tabular}{|c|c|c|c|c|c|c|c|}
\hline \multirow[b]{2}{*}{ County } & \multirow[b]{2}{*}{$\begin{array}{c}\text { State well } \\
\text { number }\end{array}$} & \multirow[b]{2}{*}{ Local well name } & \multirow{2}{*}{$\begin{array}{c}\text { Transmissivity } \\
\left(()^{2}\right) \\
\left(\mathrm{ft}^{2} / \mathrm{d}\right)\end{array}$} & \multirow{2}{*}{$\begin{array}{c}\text { Average } \\
\text { horizontal } \\
\text { hydraulic } \\
\text { conductivity } \\
\left(K_{h}\right)(\mathrm{ft} / \mathrm{d})\end{array}$} & \multirow{2}{*}{$\begin{array}{c}\text { Average } \\
\text { bulk } \\
\text { resistivity } \\
\left(R_{o}\right) \\
\text { (ohm-m) }\end{array}$} & \multicolumn{2}{|c|}{ Surface resistivity $\left(R_{s}\right)$} \\
\hline & & & & & & $\begin{array}{c}R_{s}=\left[R_{o}-R_{w}\right] \\
\quad(o h m-m)\end{array}$ & $\begin{aligned} R_{s}= & {\left[R_{o}-R_{w x}\right] } \\
& (0 \mathrm{hm}-\mathrm{m})\end{aligned}$ \\
\hline \multicolumn{8}{|c|}{ Louisiana-continued } \\
\hline Lincoln & LN-151 & Ruston Test Well & 5,880 & 45 & 60 & 36 & 36 \\
\hline Lincoln & LN-48 & Ruston Water Well \#1 & 13,400 & 130 & 150 & 110 & 110 \\
\hline Natchitoches & NA-362 & Clarence Water Well & 1,600 & 35 & 53 & 37 & 36 \\
\hline Natchitoches & NA-413 & Robeline Test Well \#2 & 590 & 18 & 25 & 17 & 17 \\
\hline Red River & RR-181 & Edgefield Test Hole \#1 & 190 & 19 & 46 & 17 & 18 \\
\hline Red River & RR-182 & Edgefield \#2 & 1,230 & 41 & 88 & 56 & 56 \\
\hline Red River & RR-241 & Choushatta Industrial Park Test \#2 & 100 & 7.1 & 26 & 13 & 12 \\
\hline Red River & RR-247 & Choushatta Test \#1 & 440 & 20 & 32 & 18 & 16 \\
\hline Red River & RR-249 & Edgefield Observation Well & 1,420 & 34 & 55 & 17 & 11 \\
\hline Red River & RR-251 & Halfway-Carroll Test \#3 & 320 & 16 & 22 & 9.1 & 7.6 \\
\hline Sabine & SA-407A & Many Test Hole \#3 & 310 & 9.7 & 23 & 15 & 15 \\
\hline Sabine & SA-407B & Many Test Hole \#3 & 930 & 47 & 55 & 38 & 36 \\
\hline Sabine & SA-431 & Toledo Bend Test Site \#4 & 230 & 11 & 25 & 13 & 12 \\
\hline Union & UN-57 & Bernice Test Well & 5,130 & 140 & 150 & 120 & 120 \\
\hline Union & UN-71B & Rocky Branch Water Test \#2 & 2,270 & 54 & 27 & 18 & 18 \\
\hline Webster & WE-139 & Louisiana Ordnance Plant & 1,260 & 19 & 32 & 17 & 16 \\
\hline Webster & WE-265 & Jenkins Water Test Well \#1 & 800 & 24 & 26 & 20 & 20 \\
\hline Webster & WE-268 & Old Shongaloo Water Well Test \#2 & 880 & 37 & 40 & 19 & 17 \\
\hline Webster & WE-270 & Jenkins Water Test Well \#2 & 630 & 24 & 34 & 25 & 24 \\
\hline Webster & WE-281 & Central Test Well \#1 & 1,120 & 40 & 38 & 24 & 23 \\
\hline Webster & WE-291 & Palmetto Beach Community \#1 & 320 & 15 & 38 & 14 & 11 \\
\hline Webster & WE-292B & North Shongaloo Community Water Well & 5,080 & 79 & 54 & 38 & 36 \\
\hline Webster & WE-308 & Thomasville Test Well \# 1 & 1,020 & 35 & 80 & 49 & 46 \\
\hline Webster & WE-321A & Germantown Water Test Well \# 1 & 7,620 & 120 & 160 & 100 & 100 \\
\hline Webster & WE-321B & Germantown Water Test Well \#1 & 3,080 & 91 & 160 & 110 & 110 \\
\hline Winn & WI-113 & Hurricane Creek \#2 & 3,610 & 52 & 34 & 26 & 25 \\
\hline Winn & WI-114 & Gum Springs Water Well Test & 4,410 & 50 & 30 & 19 & 19 \\
\hline Winn & WI-120 & Winnfield Test \#3 & 2,140 & 54 & 30 & 18 & 18 \\
\hline Winn & WI-127 & Calvin Test \#1 & 2,540 & 58 & 32 & 22 & 21 \\
\hline Winn & WI-140 & Gansville Test Well & 1,740 & 32 & 37 & 25 & 24 \\
\hline Winn & WI-143 & Calvin Test Well & 2,670 & 56 & 27 & 18 & 17 \\
\hline \multicolumn{8}{|c|}{ Mississippi } \\
\hline Calhoun & K101 & Calhoun City Well \#3 & ${ }^{4} 2,800$ & 74 & 41 & 32 & 34 \\
\hline Clarke & R31 & Hiwanee Water Assn. \#1 & ${ }^{4} 11,500$ & 89 & 35 & 23 & 25 \\
\hline Hinds & G84 & City Of Jackson "W-D" & ${ }^{4} 6,000$ & 36 & 55 & 31 & 33 \\
\hline Hinds & H146 & City Of Jackson "N-B" & ${ }^{4} 3,300$ & 30 & 78 & 52 & 54 \\
\hline Hinds & H149 & City Of Jackson & ${ }^{4} 13,000$ & 54 & 66 & 37 & 38 \\
\hline Hinds & H188 & Jackson Zoo Well \# 1 & ${ }^{4} 1,800$ & 26 & 26 & 3.7 & 4.2 \\
\hline Hinds & M99 & City Of Jackson W-B & 44,700 & 50 & 60 & 36 & 39 \\
\hline Lauderdale & $\mathrm{C} 53$ & Naval Air Station \#4 & ${ }^{4} 20,300$ & 200 & 400 & 240 & 240 \\
\hline Lauderdale & $\mathrm{C} 54$ & Naval Air Station \#1 & ${ }^{4} 21,700$ & 290 & 400 & 250 & 250 \\
\hline Lowndes & $\mathrm{P} 20$ & Weyerhaeuser \#2 & ${ }^{4} 8,700$ & 59 & 150 & 43 & 43 \\
\hline Lowndes & $\mathrm{P} 21$ & Weyerhaeuser \#3 & ${ }^{4} 5,900$ & 33 & 150 & 65 & 65 \\
\hline Madison & W74 & Town Of Madison & 44,100 & 40 & 40 & 14 & 18 \\
\hline Rankin & K119 & Town Of Pearl & ${ }^{4} 9,800$ & 47 & 40 & 13 & 16 \\
\hline Washington & L70 & Arcola Well \#3 & ${ }^{4} 28,700$ & 140 & 40 & 24 & 24 \\
\hline Yazoo & G81 & Mississippi Chemical Corp. Test Well & ${ }^{4} 18,200$ & 170 & 70 & 55 & 58 \\
\hline \multicolumn{8}{|c|}{ North Carolina } \\
\hline Jones & T27U1 & Weyerhauser Well \#2 & 2,200 & 42 & 150 & 110 & 110 \\
\hline
\end{tabular}


Table 4. Summary of borehole geoelectrical properties and aquifer hydraulic characteristics at well sites-Continued

\begin{tabular}{|c|c|c|c|c|c|c|c|}
\hline \multirow[b]{2}{*}{ County } & \multirow{2}{*}{$\begin{array}{l}\text { State well } \\
\text { number }\end{array}$} & \multirow[b]{2}{*}{ Local well name } & \multirow{2}{*}{$\begin{array}{c}\text { Transmissivity } \\
(T) \\
\left(\mathrm{ft}^{2} / \mathrm{d}\right)\end{array}$} & \multirow{2}{*}{$\begin{array}{c}\text { Average } \\
\text { horizontal } \\
\text { hydraulic } \\
\text { conductivity } \\
\left(K_{h}\right)(f t / d)\end{array}$} & \multirow{2}{*}{$\begin{array}{c}\text { Average } \\
\text { bulk } \\
\text { resistivity } \\
\left(R_{o}\right) \\
\text { (ohm-m) }\end{array}$} & \multicolumn{2}{|c|}{ Surface resistivity $\left(R_{s}\right)$} \\
\hline & & & & & & $\begin{array}{c}R_{s}=\left[R_{0}-R_{w}\right] \\
\quad(0 h m-m)\end{array}$ & $\begin{array}{c}R_{s}=\left[R_{o}-R_{w x}\right. \\
\quad(o h m-m)\end{array}$ \\
\hline \multicolumn{8}{|c|}{ South Carolina } \\
\hline Barnwell & BW-79 & Town Of Williston & ${ }^{5} 13,000$ & 39 & 270 & 86 & 65 \\
\hline Dorchester & DOR-221 & Oakbrook Well \#3 & 590 & 9.2 & 11 & 0.8 & 2.0 \\
\hline Horry & HO-336 & North Myrtle Beach \#2 & ${ }^{5} 2,000$ & 6.3 & 7.8 & 2.4 & 2.6 \\
\hline Horry & $\mathrm{HO}-416$ & Ocean Lakes \#6 & ${ }^{5} 2,700$ & 7.0 & 17 & 7.7 & 7.5 \\
\hline \multicolumn{8}{|c|}{ Tennessee } \\
\hline Lauderdale & LD:H-6 & City Of Ripley \#2 & 22,300 & 250 & 200 & 150 & 140 \\
\hline Shelby & SH:J-104 & Memphis Light, Gas, and Water & 19,800 & 280 & 300 & 230 & 220 \\
\hline Shelby & SH:K-73 & Memphis Light, Gas, and Water Test \#6 & 21,100 & 160 & 380 & 310 & 290 \\
\hline Shelby & SH:L-69 & Memphis Light, Gas, and Water & 26,700 & 240 & 400 & 290 & 260 \\
\hline Shelby & SH:L-8 & Germantown Well \#2 & 21,200 & 210 & 550 & 390 & 360 \\
\hline Shelby & SH:P-25 & Buckeye \#10 & 14,100 & 72 & 190 & 130 & 130 \\
\hline Shelby & SH:U-18 & Dupont Well \#5 & 56,800 & 560 & 160 & 100 & 97 \\
\hline
\end{tabular}

${ }^{1}$ Davis and others (1983).

${ }^{2}$ Scott and others (1984).

${ }^{3}$ Faye and McFadden (1986).

${ }^{4}$ Slack and Darden (1991).

${ }^{5}$ Aucott and Newcome (1986).

\section{RELATIONS OF BOREHOLE RESISTIVITY TO AQUIFER HYDRAULIC CHARACTERISTICS AND WATER QUALITY}

Relations of geoelectrical properties to hydraulic and water-quality characteristics were developed using the general logarithmic regression model

$$
Y=A X^{B},
$$

where $Y$ and $X$ are dependent and independent variables, respectively, and $A$ and $B$ are regression coefficients. This model has previously been used by investigators to evaluate the relations of aquifer characteristics to geoelectrical properties (Alger, 1966; Mazac and others, 1985; Huntley, 1986; and Yao An Guo, 1986).

Measurements of bulk resistivity and horizontal hydraulic conductivity require an evaluation of the aquifer interval contributing water to a well (table 1). Contributing intervals were determined by comparing reported screened intervals to the thickness of juxtaposed sands and clayey sands identified on lithologic and corresponding borehole geophysical logs. Contributing intervals were commonly bounded above and below by clays of considerable thickness (fig. 2). In general, the estimated contrib- uting interval for large-capacity wells (discharge greater than 500 gallons per minute ( $\mathrm{gal} / \mathrm{min})$ ) was somewhat to substantially larger than the screened interval. Where screened intervals were small compared to the total thickness of a water-bearing unit and well discharges were also comparatively small (ranging from 20 to $50 \mathrm{gal} / \mathrm{min}$ ), contributing intervals and screened intervals frequently were considered coincident or nearly coincident.

Infrequently, large disparities occurred between the total screen length at a well and the total estimated contributing interval. At these sites, short-interval screens commonly were placed opposite relatively thick water-bearing sands. Because the contribution of each sand interval to total well discharge was unknown, the total sand thickness of partially screened intervals was considered the contributing interval. Estimates of total contributing interval at wells used in this study ranged from 10 to $630 \mathrm{ft}$ and were considered to be accurate within \pm 25 percent of reported values (table 1 ).

Aquifer bulk resistivity for this study was considered to approximately equal resistivity measured by the long-normal (64-inch) resistivity log or by the deep induction log. Long-normal logs were unavailable at sites 31Z002, in Burke County, Ga., SH:P-25 in Shelby County, Tenn., and SRP 
Table 5. Summary of results of regression analyses

[DSC, dissolved-solids concentration; $F a$, apparent formation factor; $K_{h}$, horizontal hydraulic conductivity; $R_{o}$, bulk resistivity; $R_{w x}$, water resistivity; LC, Late Cretaceous; PLE, Paleocene-early Eocene; ME, middle Eocene] (General equation $Y=A X^{B}$ )

\begin{tabular}{llccccc}
\hline $\begin{array}{c}\text { Dependent } \\
\text { variable } \\
Y\end{array}$ & $\begin{array}{c}\text { Independent } \\
\text { variable } \\
X\end{array}$ & $\begin{array}{c}\text { Coefficient } \\
A\end{array}$ & $\begin{array}{c}\text { Exponent } \\
B\end{array}$ & $\begin{array}{c}\text { Correlation } \\
\text { coeficient } \\
\text { (percent) }\end{array}$ & $\begin{array}{c}\text { Standard } \\
\text { error of } \\
\text { estimate } \\
\text { (log cycle) }\end{array}$ & $\begin{array}{c}\text { Number } \\
\text { of data } \\
\text { pairs }\end{array}$ \\
\hline$K_{h}$ & $\left(R_{o}-R_{w x}\right)$ & 3.8 & 0.66 & 72 & 0.68 & 105 \\
$K_{h}$ & $F a$ & 14 & 1.0 & 38 & .91 & 106 \\
$K_{h}$ & $R_{w x}$ & 5.7 & .59 & 56 & .82 & 106 \\
$K_{h}$ & $R_{o}$ & 1.8 & .74 & 70 & .70 & 105 \\
$R_{w x}$ & $R_{o}$ & .56 & .92 & 93 & .35 & 111 \\
$D S C$ & $R_{o}$ & 8,500 & -.85 & -88 & .44 & 106 \\
$D S C$ & $R_{w x}$ & 5,110 & -.92 & -96 & .27 & 106 \\
$K_{h}(\mathrm{LC})$ & $\left(R_{o}-R_{w x}\right)$ & 5.5 & .43 & 80 & .52 & 14 \\
$K_{h}(\mathrm{PLE})$ & $\left(R_{o}-R_{w x}\right)$ & 1.5 & .88 & 78 & .56 & 47 \\
$K_{h}(\mathrm{ME})$ & $\left(R_{o}-R_{w x}\right)$ & 8.9 & .54 & 71 & .56 & 44 \\
$K_{h}(\mathrm{LC})$ & $R_{o}$ & 3.2 & .48 & 77 & .55 & 14 \\
$K_{h}(\mathrm{PLE})$ & $R_{o}$ & .57 & 1.0 & 78 & .56 & 47 \\
$K_{h}(\mathrm{ME})$ & $R_{o}$ & 3.8 & .67 & 71 & .56 & 44 \\
\hline
\end{tabular}

905-120p in Barnwell County, S.C., and resistivity measured by the short-normal (16-inch) log was used to estimate bulk resistivity. Average bulk resistivity for a contributing interval was determined by computing the area between the log trace and the line of zero resistivity and dividing by the respective vertical interval. Resistivity $\log$ scales varied throughout the study area. In general, vertical scale resolution was larger than $100 \mathrm{ft}$ per inch and horizontal scale resolution was larger than $200 \mathrm{ohm}-\mathrm{m}$ per inch.

The formation temperature was considered equal to water temperature at the point of well discharge and was available at most well sites. Watertemperature measurements were considered accurate within $\pm 0.5^{\circ} \mathrm{C}$ (table 3 ).

Specific conductance determined by laboratory or field measurements is commonly reported in micromhos or microsiemens per centimeter at a standard temperature of $25^{\circ} \mathrm{C}\left(77^{\circ} \mathrm{F}\right)$; therefore, it is related to aquifer water resistivity at $77^{\circ} \mathrm{F}$ in ohm-m, by the following expression (Miller and others, 1988),

$$
R_{w 77}=10,000 / \text { specific conductance. }
$$

Where water discharge temperatures were known (table 3), aquifer water resistivity $\left(R_{w}\right)$ was converted from resistivity at the standard temperature of $77^{\circ} \mathrm{F}$ to the resistivity at the known temperature $\left(R_{w x}\right)$. This conversion was accomplished using the equation described by Jorgensen (1989),

$$
R_{w x}=\frac{R_{w 77} \times 84}{T_{x}+7}
$$

where

$R_{w x}$ is aquifer water resistivity at the observed water temperature at the point of well discharge, in ohm-m;

$R_{w 77}$ is aquifer water resistivity at the standard temperature, in ohm-m; and

$T_{x}$ is water temperature at the point of well discharge, in degrees Fahrenheit.

Where well discharge temperatures were unknown, aquifer water resistivities were unadjusted and based on the standard temperature of $77^{\circ} \mathrm{F}$. Aquifer water resistivity measurements at standard temperature are considered accurate within \pm 10 percent of reported values (table 3 ).

Computed values of horizontal hydraulic conductivity used in regression analyses (figs. 3-6, table 5) represent average aquifer transmissivity per unit length of contributing interval (table 1). Transmissivity values used to compute horizontal hydraulic conductivity were derived, for the most part, from analyses of single-well, aquifer-test data using modified nonequilibrium methods (Ferris and others, 1962). A few results of multiple-well, nonequilibrium analyses (Theis, 1935; Hantush and Jacob, 1955) were also used.

Uncertainty related to determinations of contributing interval, bulk resistivity, water resistivity, and horizontal hydraulic conductivity can be attributed to random and systematic errors of measurement, and to spatial variations of geoelectrical prop- 


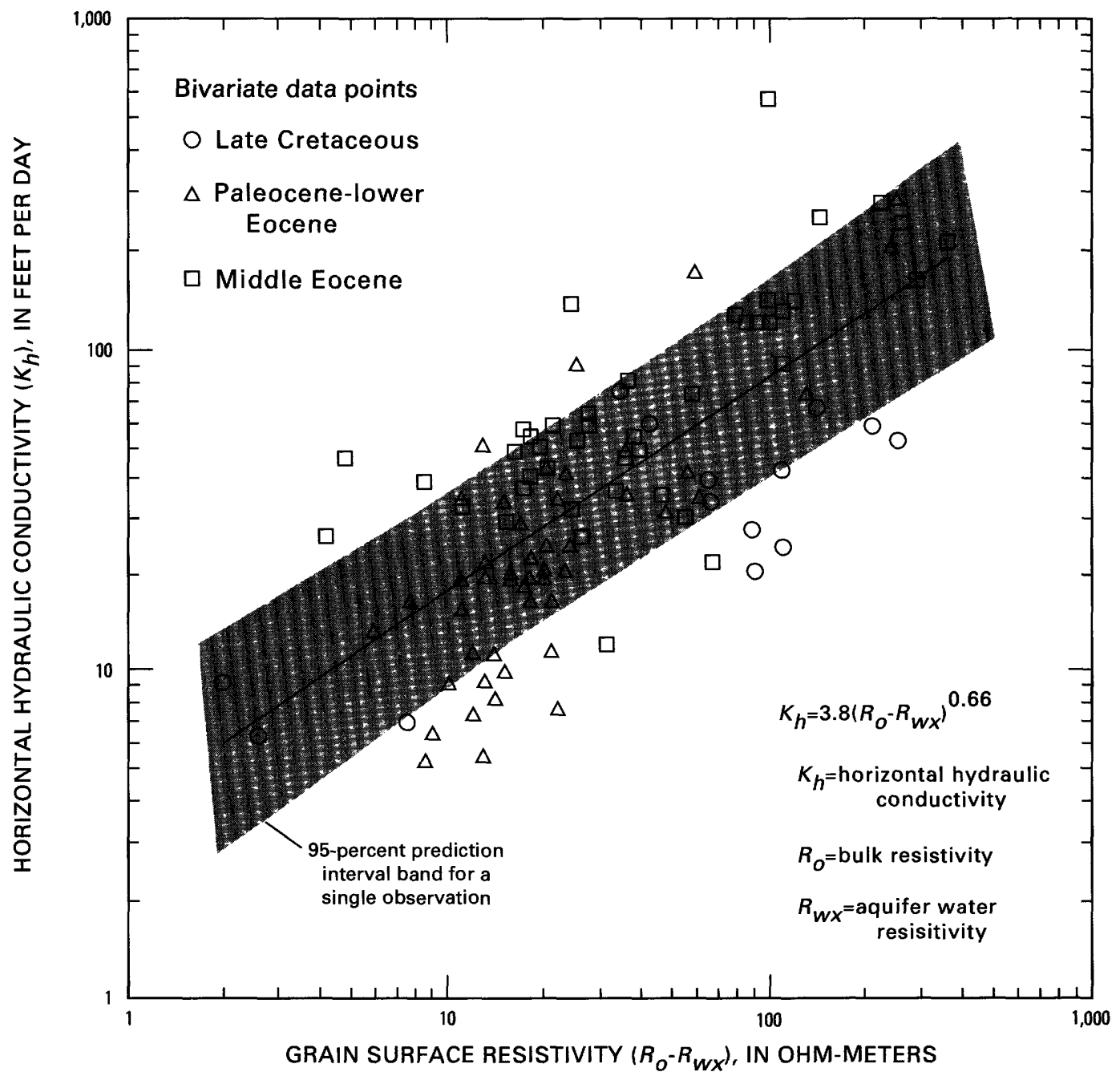

Figure 3. Relation of honizontal hydraulic conductivity to grain-surface resistivity as defined by the summation model.

erties and hydraulic characteristics. Measurement errors relate largely to inaccuracies caused by field and laboratory instruments or observations and could only be indirectly evaluated for this study. For example, site data were eliminated from further analyses where aquifer water resistivity exceeded average bulk resistivity. Such anomalies were reported infrequently and were attributed to instrument error.

Uncertainties related to transmissivity evaluations are probably the result of errors introduced by the spatial variation of aquifer hydraulic characteristics. Standard methods of analysis for transmissivity are generally based on assumptions that aquifer properties are spatially constant. Such assumptions are seldom, if ever, completely satisfied by waterbearing units. Regardless, standard methodologies of aquifer-test analysis have consistently been applied in the study area with apparently successful results (Hosman and others, 1968; Zack, 1977; Davis and others, 1983; Faye and McFadden, 1986; Slack and Darden, 1991) and transmissivity values reported here are considered equally valid (table 4). To account for uncertainty, computed transmissivity values are considered accurate within \pm 25 percent of reported values (table 4).

Differences in aquifer volume represented by borehole resistivity measurements and aquifer-test results may be substantial at large-capacity wells. Lateral variation in the hydraulic characteristics and geoelectrical properties of local water-bearing units is, however, probably small within the radius of influence of a pumping well. Thus, geoelectrical properties and hydraulic characteristics determined 


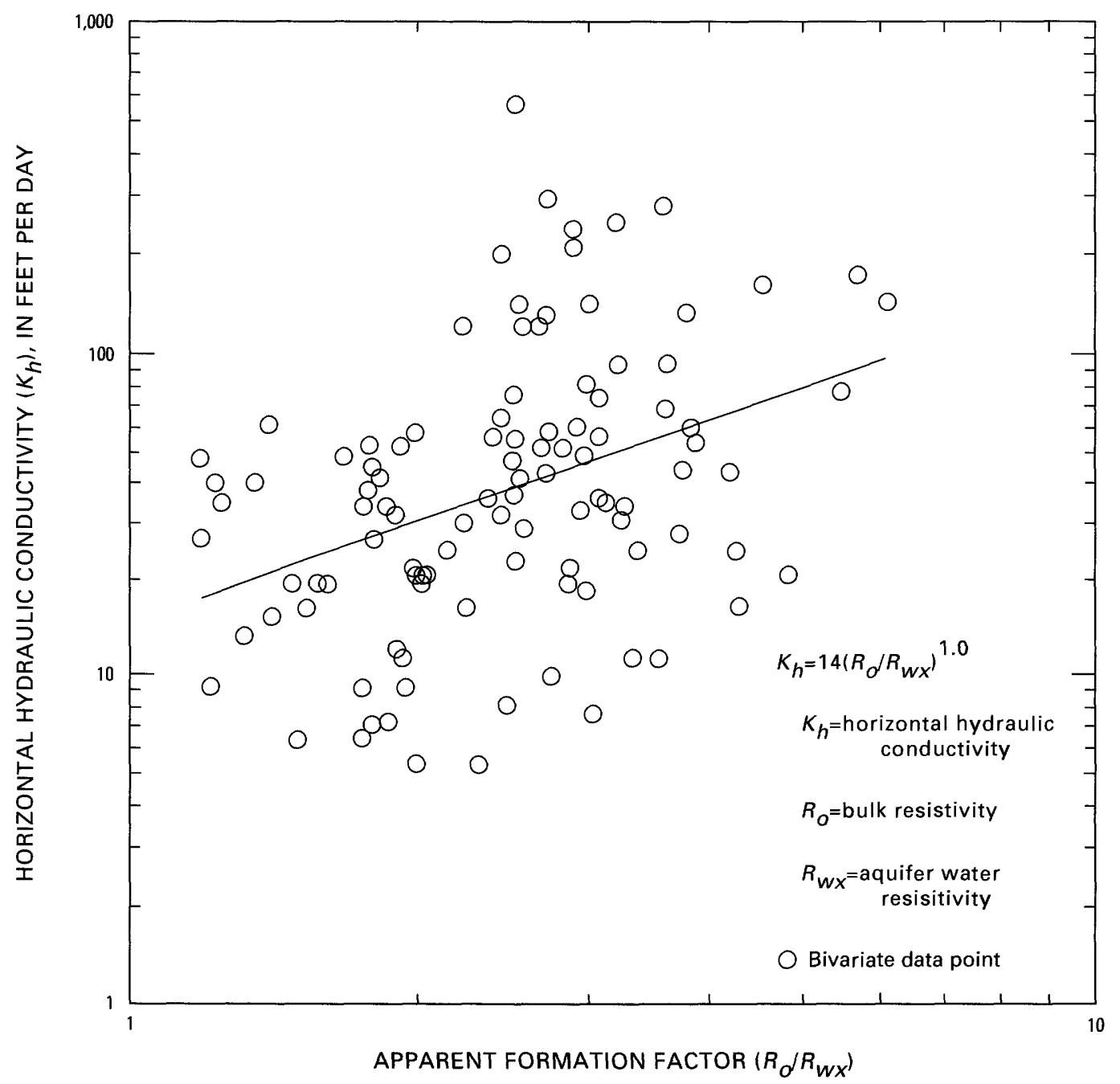

Figure 4. Relation of horizontal hydraulic conductivity to apparent formation factor as defined by the product model.

from substantially different aquifer volumes are considered representative of the entire aquifer volume contributing water to the well. To ensure that average hydraulic conductivity values were based on a representative aquifer volume, data from aquifer tests of short duration (less than 2 hours) were not used. Aquifer-test duration related to transmissivity data cited in this report exceeded 4 hours at most sites (table 4; Aucott and Newcome, 1986; Faye and McFadden, 1986; Slack and Darden, 1991).

Mud invasion of permeable zones during drilling also can substantially affect borehole resistivity and aquifer-test results. Where substantial invasion of drilling mud has occurred, resistivity measurements will reflect the geoelectrical properties of the drilling mud, rather than of the aquifer. In addition, analyses of aquifer-test data collected by pumping from mud-invaded sands generally result in computed transmissivities that are lower than corresponding values for contiguous noninvaded sands. Mud-invaded zones were identified by comparing the divergence between short- and long-normal resistivity measurements (fig. 2). Where invasion occurred across a substantial part of the contributing interval and well development appeared limited or ineffective, site data were rejected for further analyses.

Regression analyses using equation 9 were completed for various combinations of geoelectrical, hydraulic, and water-quality data listed in tables 3 and 4. Results of these analyses are summarized below (eqs. 12-17), in table 5, and in figures 3-9. Discharge temperature data were unavailable at 22 sites (table 3). Accordingly, water resistivity at 


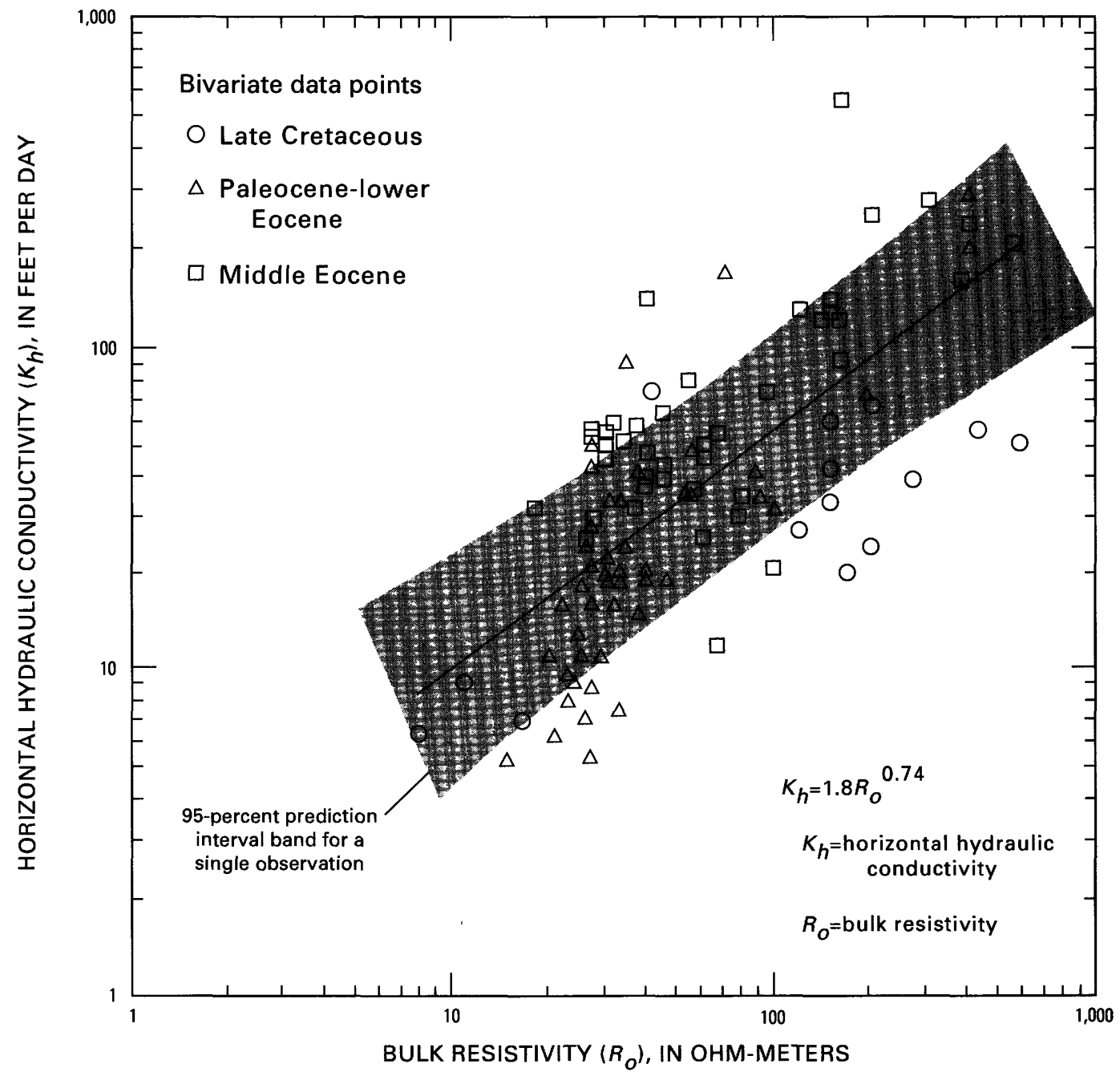

Figure 5. Relation of horizontal hydraulic conductivity to aquifer bulk resistivity.

these sites used in regression analyses (eqs. 13, 16) were standard $\left(R_{w 77}\right)$ rather than adjusted $\left(R_{w x}\right)$. Correlation coefficients for all analyses range from about 70 to 96 percent, except for the product model relation of horizontal hydraulic conductivity to apparent formation factor, which is $\mathbf{3 8}$ percent (table 5). These results, when compared to the results of regression analyses based on the summation model (eq. 12) and aquifer bulk resistivity (eq. 17), indicate that the apparent formation factor (eq. 8 ) is not a useful geoelectrical analog for the horizontal hydraulic conductivity of clastic aquifers considered in this study. Accordingly, references in this report to relations of horizontal hydraulic conductivity to grain-surface resistivity refer only to the relation explained by the summation model (eq. 7).
Following are the regression equations that relate horizontal hydraulic conductivity $\left(K_{h}\right)$, dissolved-solids concentrations $(D S C)$ in aquifer water, and aquifer water resistivity $\left(R_{w x}\right)$ to the borehole geoelectrical properties of bulk resistivity $\left(R_{o}\right)$ and grain-surface resistivity (defined by the summation model $\left.\left(R_{o}-R_{w x}\right)\right)$ :

$$
\begin{gathered}
K_{h}=3.8\left(R_{o}-R_{w x}\right)^{0.66}, \\
D S C=5110 R_{w x}^{-0.92}, \\
D S C=8500 R_{o}^{-0.85}, \\
R_{w x}=0.56 R_{o}^{0.92}, \\
K_{h}=5.7 R_{w x}^{0.59}, \text { and } \\
K_{h}=1.8 R_{o}^{0.74} .
\end{gathered}
$$




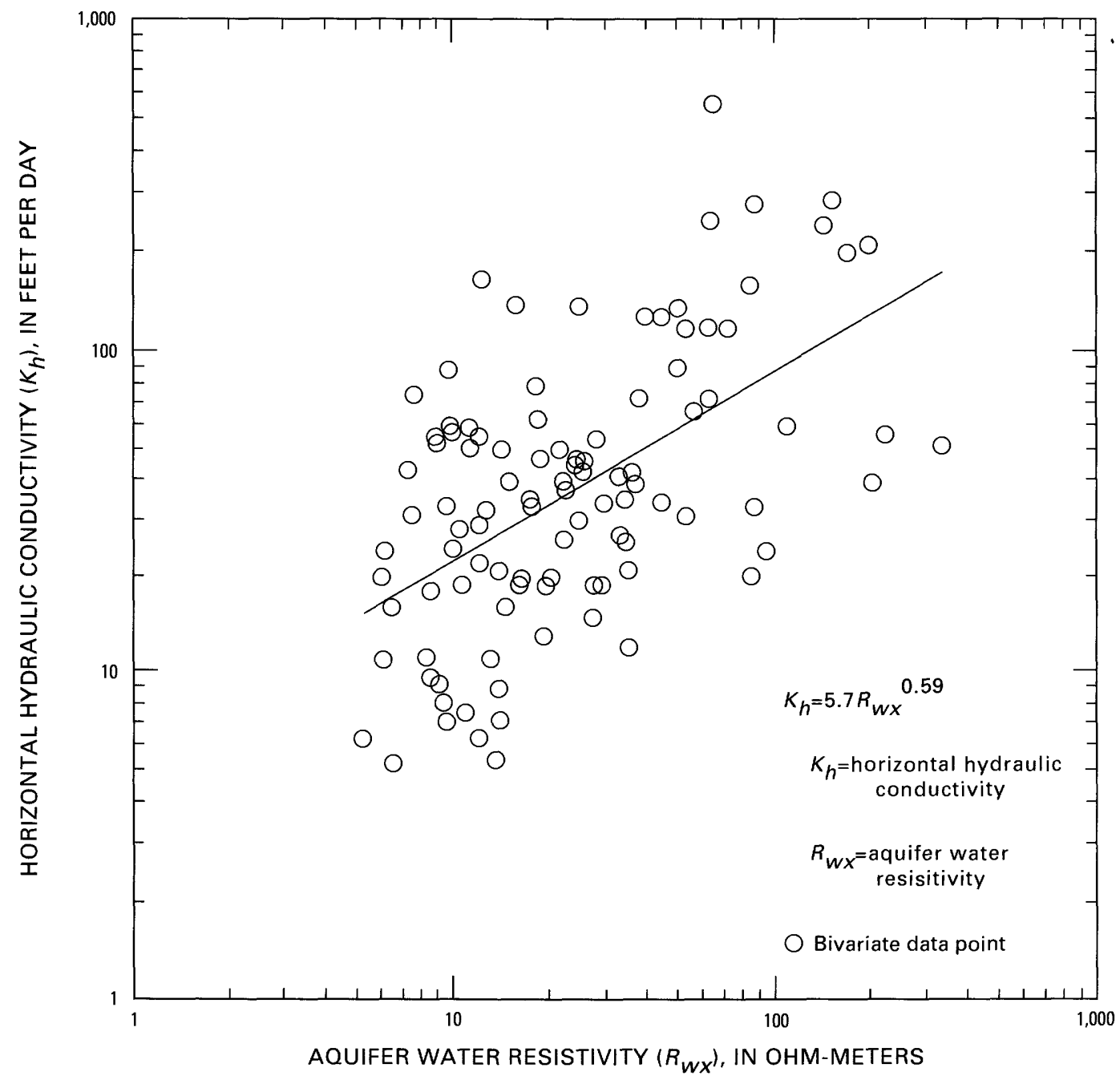

Figure 6. Relation of horizontal hydraulic conductivity to aquifer water resistivity.

Regression statistics for equations 12 and 17 (table 5) indicate that grain-surface resistivity as defined by the summation model (eq. 7) and bulk resistivity $\left(R_{o}\right)$ are equally significant geoelectrical analogs for horizontal hydraulic conductivity $\left(K_{h}\right)$.

Quasi-validation of the use of grain-surface resistivity as a geoelectrical analog for horizontal hydraulic conductivity is indicated by the regression analysis relating horizontal hydraulic conductivity $\left(K_{h}\right)$ to aquifer water resistivity $\left(R_{w x}\right.$, table 5 , fig. $6)$. Consider that the grain-surface resistivity of a freshwater-saturated, unconsolidated, clastic porous media decreases as the percentage (and surface area) of fine-grained sediments increases. Such increases might be indicated by corresponding increases in the ionic strength of the aquifer water and in the ion exchange between the water and fine-grained sediments, particularly clays (Alger, 1966). Accord- ingly, a positive trend should be evident between horizontal hydraulic conductivity and the resistivity of aquifer water. Although regression statistics are not strongly conclusive (table 5), increasing values of aquifer water resistivity are shown to generally relate to increasing values of horizontal hydraulic conductivity.

Evaluation of figures 3 and 5 indicates that a general grouping of hydraulic conductivity data occurs based on the age of aquifer sediments; the lowest values generally relate to aquifers composed of sediments of Cretaceous age and the highest values generally correspond to aquifers composed of sediments of middle Eocene age. Thus, horizontal hydraulic conductivity and estimated values of grain-surface resistivity paired according to site and age were related using the logarithmic regression model (eq. 9). Data were grouped into three major 


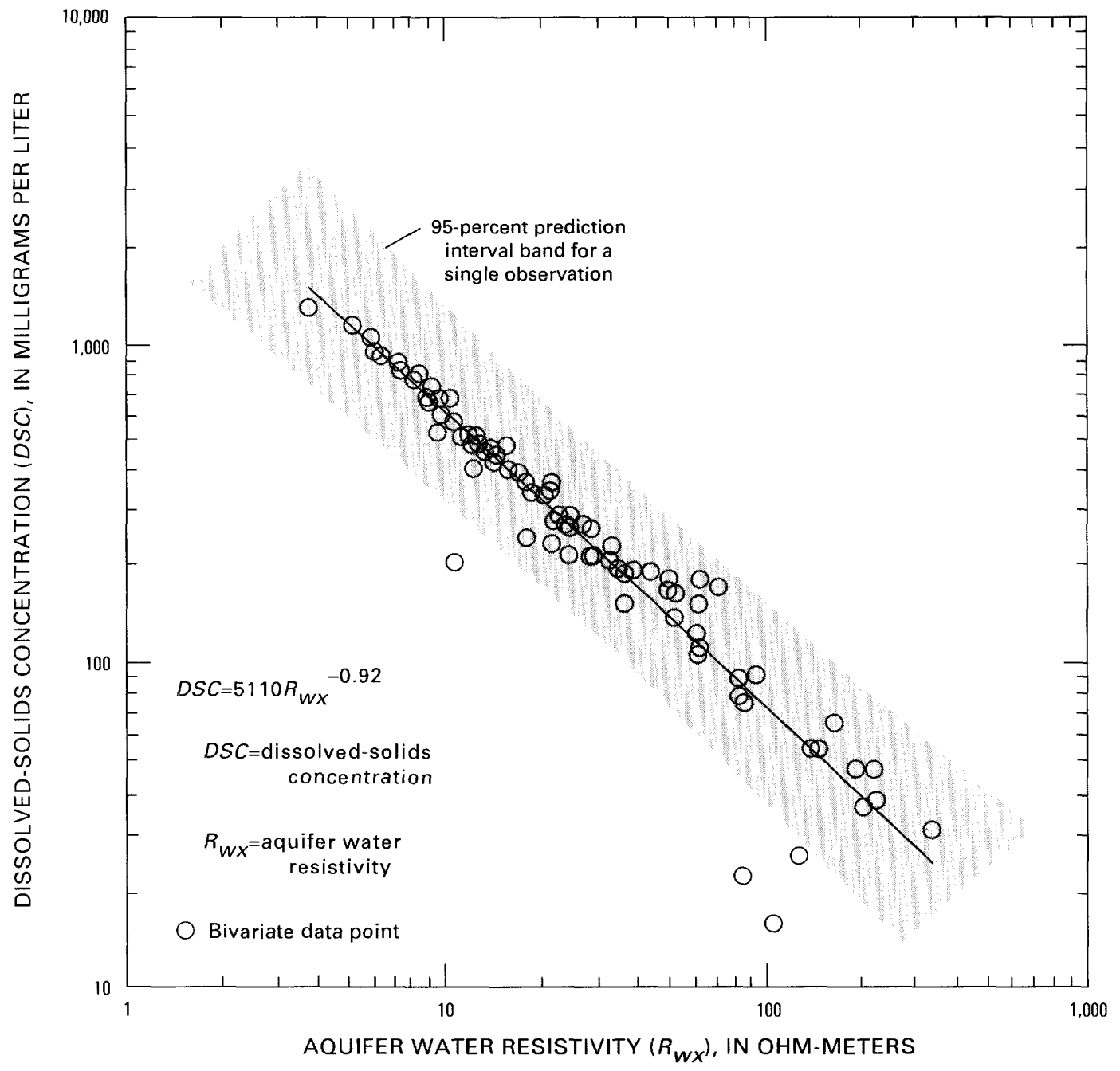

Figure 7. Relation of dissolved-solids concentration in aquifer water to aquifer water resistivity.

chronostratigraphic groups corresponding to aquifers composed of sediments of Late Cretaceous, Paleocene and early Eocene, and middle Eocene age.

Regression equations describing these relations using surface resistivity $\left(R_{o}-R_{w x}\right)$ as the independent variable are

Late Cretaceous

Paleocene and

$$
K_{h}=5.5\left(R_{o}-R_{w x}\right)^{0.43}
$$

early Eocene

$$
K_{h}=1.5\left(R_{o}-R_{w x}\right)^{0.88}, \text { and }
$$

middle Eocene

$$
K_{h}=8.9\left(R_{o}-R_{w x}\right)^{0.54} \text {. }
$$

Corresponding equations using bulk resistivity $\left(R_{o}\right)$ as the independent variable are

Late Cretaceous

$$
K_{h}=3.2 R_{o}{ }^{0.48},
$$

Paleocene and early Eocene

$$
K_{h}=0.57 R_{o}{ }^{1.0} \text {, and }
$$

middle Eocene

$$
K_{h}=3.8 R_{o}^{0.67} \text {. }
$$

Regression statistics for these equations indicate a moderate degree of correlation between the independent and dependent variables. Correlation coefficients range from 71 to 80 percent and standard errors of estimate range from 0.52 to $0.56 \mathrm{log}$ cycles (table 5). Data pairs related to 14 sites were used to develop equations 18 and 21 . These data were obtained from South Carolina, Georgia, and Mississippi. The number of site data pairs used to develop equations 19 and 22, and 20 and 23 are, respectively, 47 and 44 (table 5). Data related to equations 20 and 23 are the most widespread in the study area, ranging from Georgia to western Tennessee. 


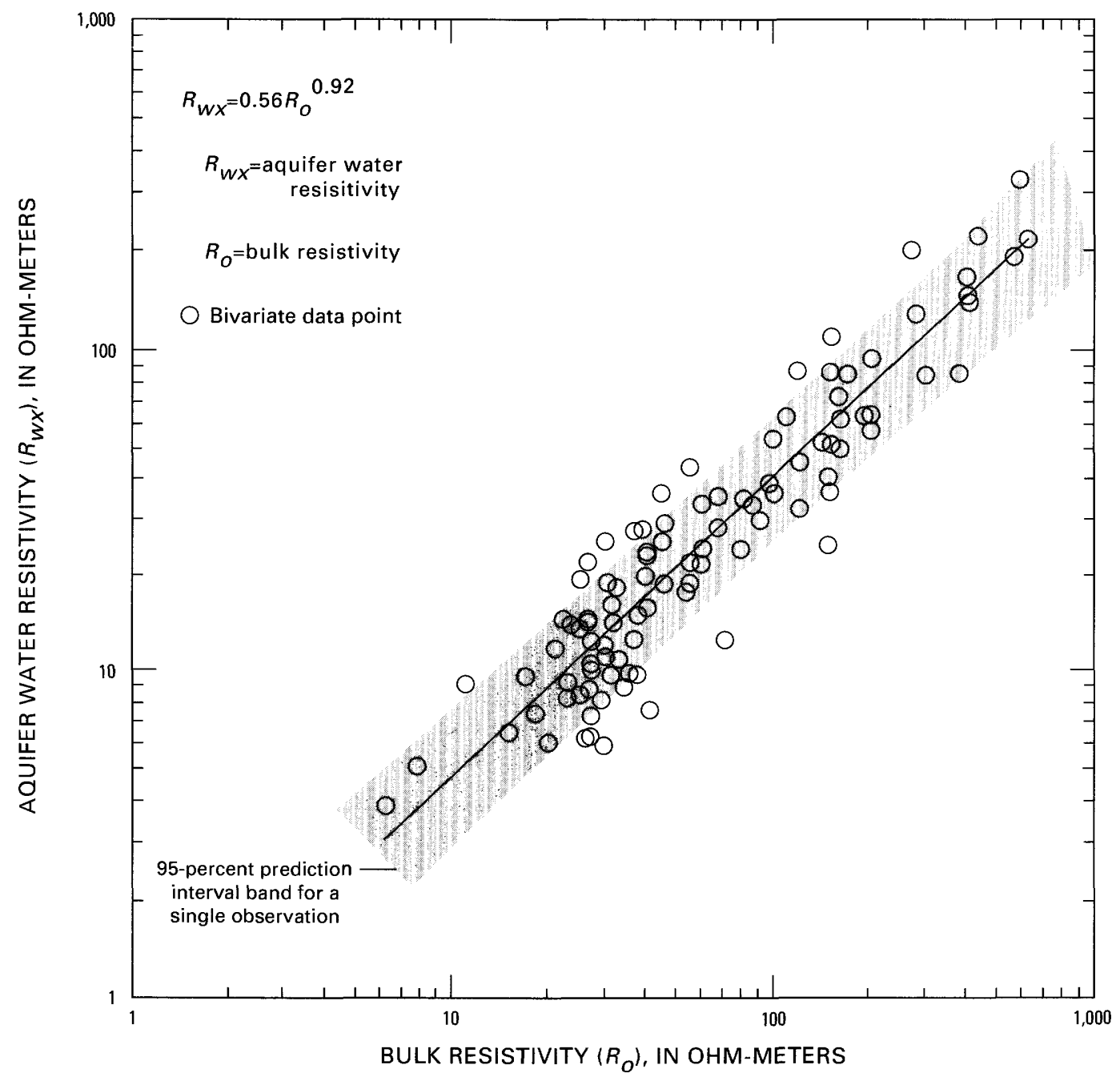

Figure 8. Relation of aquifer water resistivity to aquifer bulk resistivity.

\section{APPLICATIONS AND TESTING OF REGRESSION MODELS}

Equations $12-15$ and $17-23$ can be used to provide estimates of the vertical distribution of aquifer horizontal hydraulic conductivity $\left(K_{h}\right)$, aquifer water resistivity $\left(R_{w x}\right)$, and dissolved-solids concentration $(D S C)$ of aquifer water at borehole sites in the study area. Applications should be limited to aquifers in the Southeastern United States consisting of unconsolidated sands and clayey sands ranging in age from Late Cretaceous to middle Eocene. Equations relating horizontal hydraulic conductivity to geoelectrical properties may not be valid for sand thicknesses less than $10 \mathrm{ft}$ or for sands containing saline water. Specifically, the regression equations should not be applied to water-bearing sands where hydraulic and water-quality characteristics are substantially different from those used to develop the regression relations. In the absence of water-quality data, equations 15,12 , and 14 can be applied sequentially to provide estimates of aquifer water resistivity, horizontal hydraulic conductivity, and dissolved-solids concentration. All applications, with the exception of equation 13 , require the use of reasonable surrogates of aquifer bulk resistivity, such as long-normal (64-inch) resistivity.

Regression equations 13-15 and 18-23 (tables 6-9) were applied to bivariate data from selected well sites in the Southeastern United States. Equations 13-15 were applied at several borehole sites, largely in South Carolina, where measurements of the specific conductance and dissolved-solids concentrations of aquifer water are reported at several depths (Lee, 1984). 


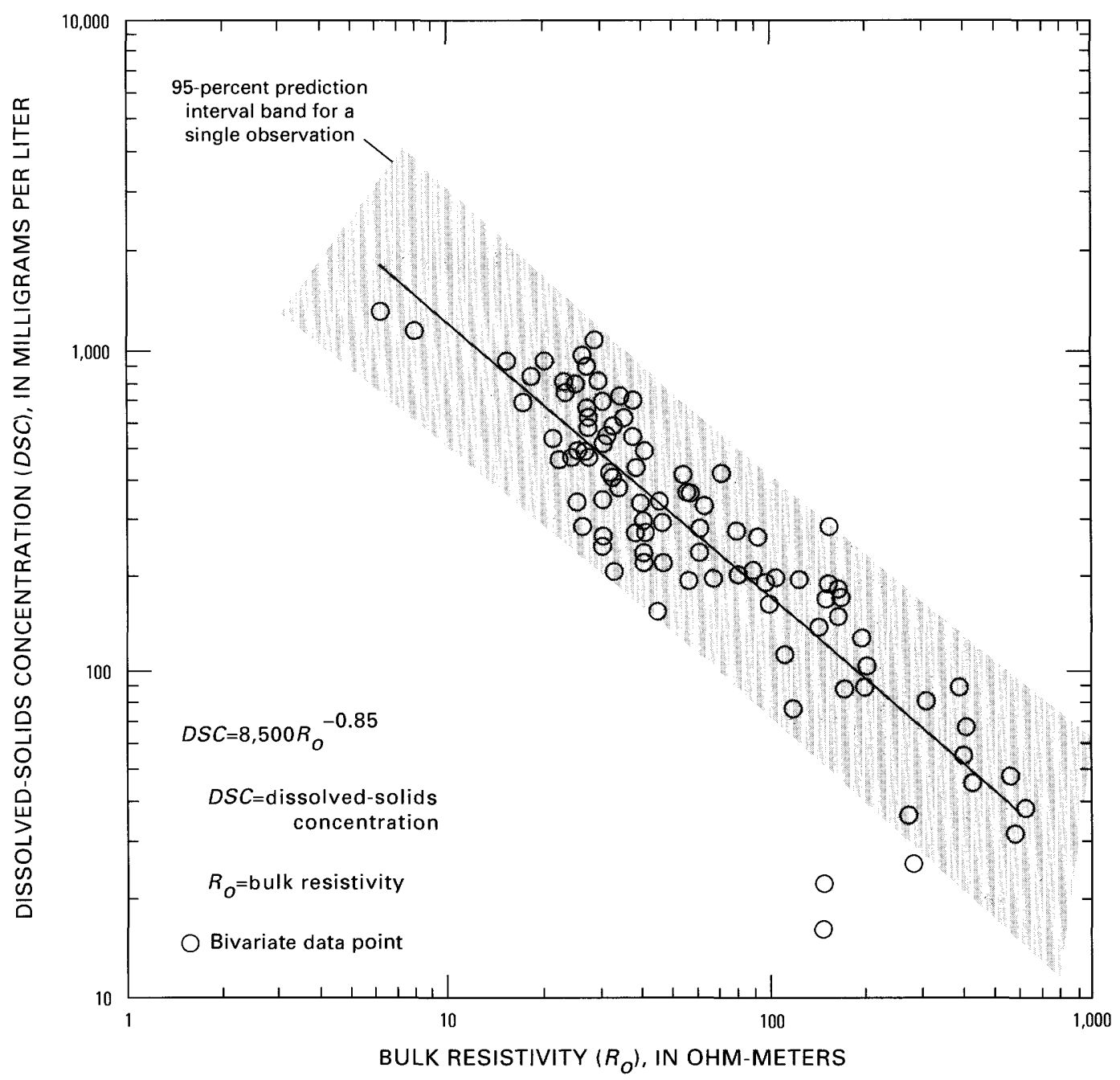

Figure 9. Relation of dissolved-solids concentration in aquifer water to aquifer bulk resistivity.

Equations 18, 19, 21, and 22 were applied at site CH-186, near Charleston, S.C. (table 1), where borehole geophysical logs, well construction information, aquifer hydraulics data, and water-quality data permit the computation of horizontal hydraulic conductivity using estimates of both grain-surface resistivity and bulk resistivity. Horizontal hydraulic conductivity values computed using regression models were compared to corresponding values determined from a single aquifer test using the completed well, and from laboratory permeameter analyses of core samples. Equations 21-23 were used to estimate horizontal hydraulic conductivity at 26 other sites where water resistivity data were unavailable.

The application of regression equations 12-15 and 17-23 indicates that aquifer water resistivity, dissolved-solids concentration, and horizontal hydraulic conductivity can be estimated with reasonable accuracy using appropriate geoelectrical analogs and regression-based models. Observed dissolvedsolids concentrations at a total of four sites in South Carolina and Louisiana ranged from about 300 to $1,300 \mathrm{mg} / \mathrm{L}$ and were compared to estimates computed using bulk resistivity and aquifer water resistivity as independent variables (table 6). Estimates of dissolved-solids concentration based on aquifer water resistivity (eq. 13) were generally more accurate (particularly at high concentrations of dissolved solids) than estimates based on bulk resistivity (eq. 14), with mean absolute errors of 6.2 and 20 percent, respectively (table 8). Estimates of aquifer water resistivity based on bulk resistivity (eq. 15) 


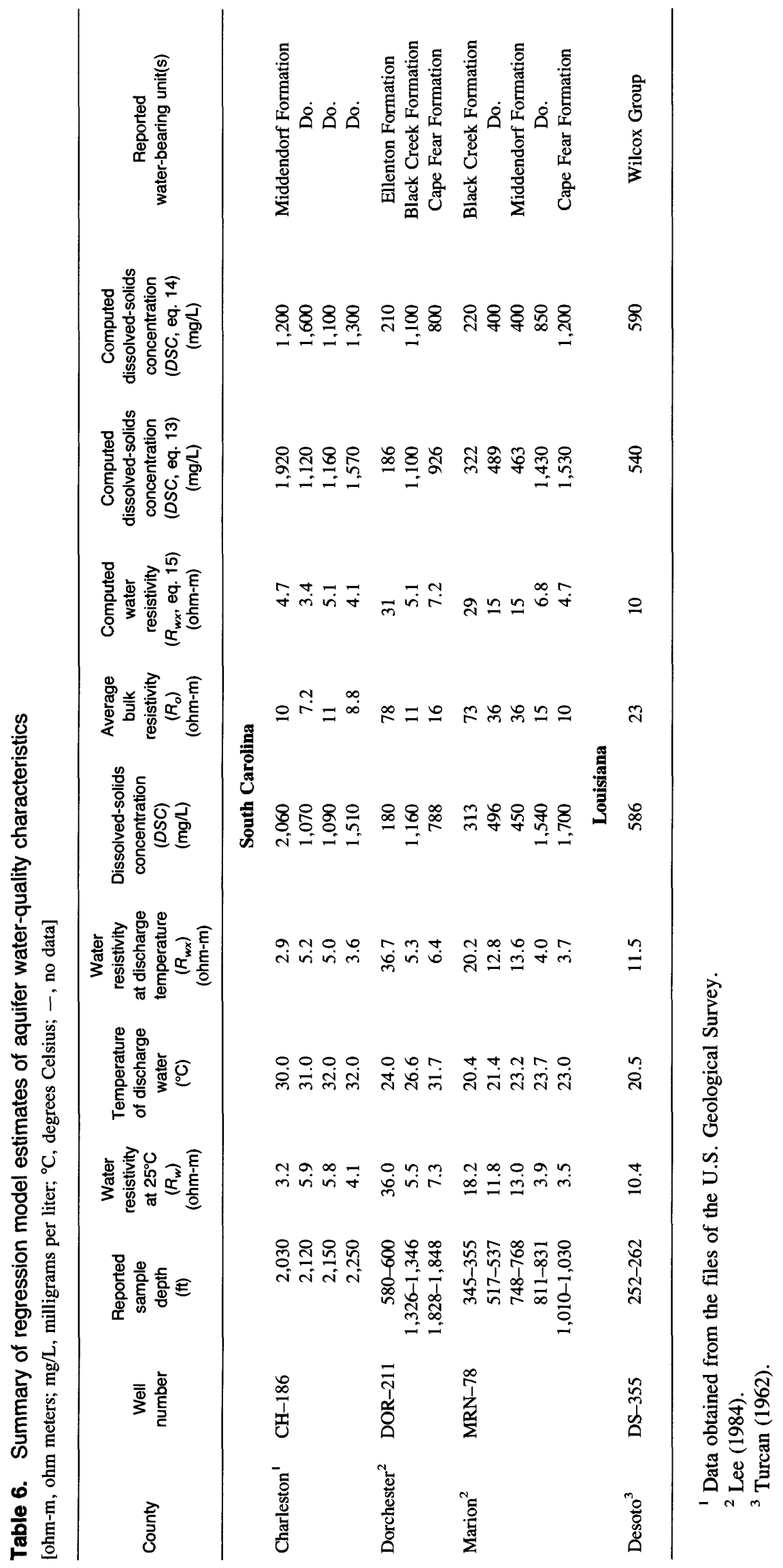




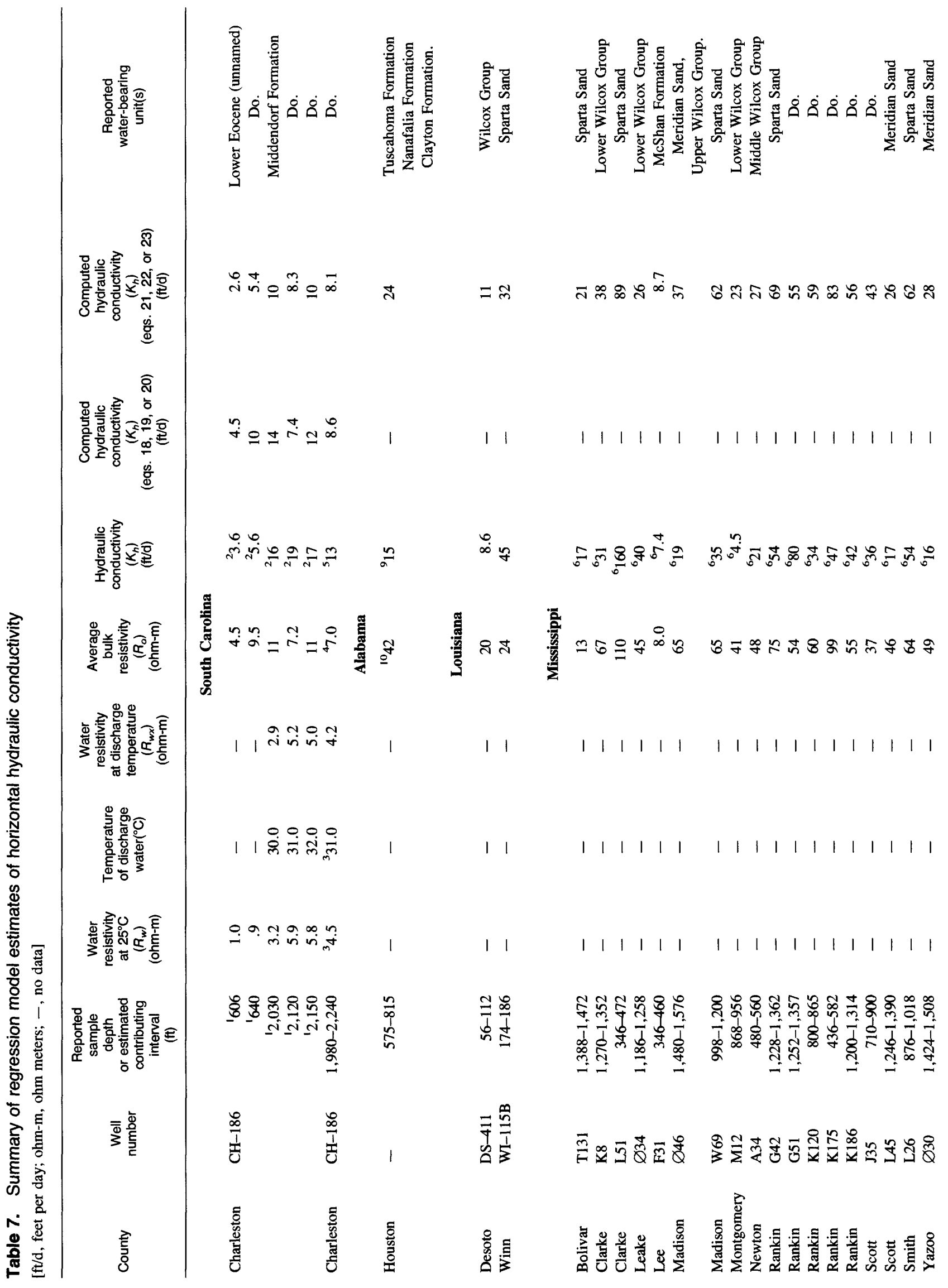




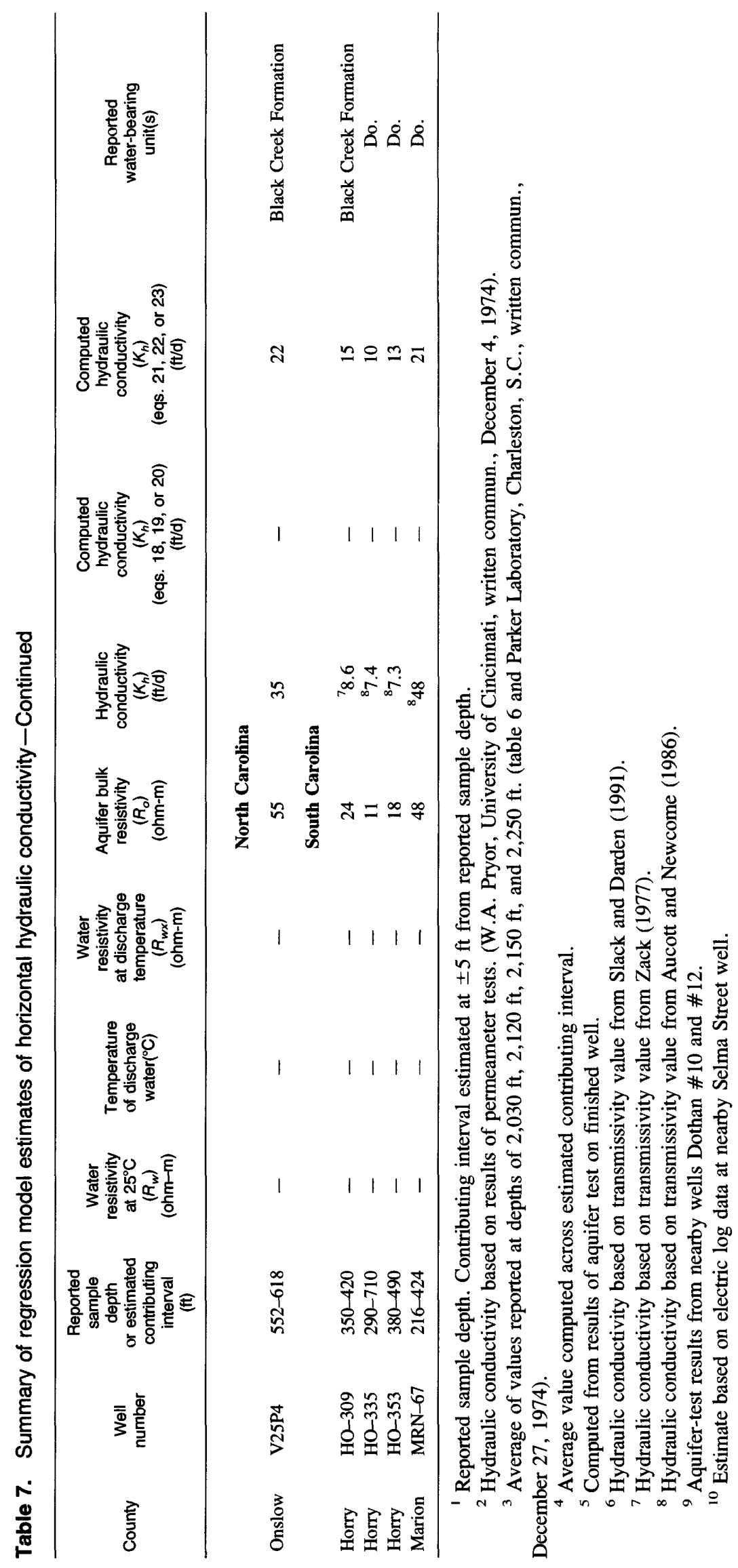


Table 8. Summary of error analyses of regression model estimates of aquifer water-quality characteristics

[DSC, dissolved-solids concentration; $R_{w x}$, water resistivity; $R_{o}$, bulk resistivity]

\begin{tabular}{lcccccc}
\hline $\begin{array}{c}\text { Dependent } \\
\text { variable }\end{array}$ & $\begin{array}{c}\text { Independent } \\
\text { variable }\end{array}$ & $\begin{array}{c}\text { Equation } \\
\text { number }\end{array}$ & $\begin{array}{c}\text { Number } \\
\text { of } \\
\text { estimates }\end{array}$ & $\begin{array}{c}\text { Range of } \\
\text { estimate error } \\
\text { (percent) }\end{array}$ & $\begin{array}{c}\text { Mean } \\
\text { estimate error } \\
\text { (percent) }\end{array}$ & $\begin{array}{c}\text { Standard deviation } \\
\text { of estimate error } \\
\text { (percent) }\end{array}$ \\
\hline$D S C$ & $R_{w x}$ & 13 & 13 & $1.4-18$ & 6.2 & 4 \\
$D S C$ & $R_{o}$ & 14 & 13 & $.7-50$ & 20 & 17 \\
$R_{w x}$ & $R_{o}$ & 15 & 13 & $1.9-70$ & 25 & 21 \\
\hline
\end{tabular}

Table 9. Summary of error analyses of regression model estimates of horizontal hydraulic conductivity

[ $K_{h}$, horizontal hydraulic conductivity; $R_{o}$, bulk resistivity; $R_{w x}$, water resistivity]

\begin{tabular}{lcrcccc}
\hline $\begin{array}{c}\text { Dependent } \\
\text { variable }\end{array}$ & $\begin{array}{c}\text { Independent } \\
\text { variable }\end{array}$ & $\begin{array}{c}\text { Equation } \\
\text { number }\end{array}$ & $\begin{array}{c}\text { Number } \\
\text { of } \\
\text { estimates }\end{array}$ & $\begin{array}{c}\text { Range of } \\
\text { estimate error } \\
\text { (percent) }\end{array}$ & $\begin{array}{c}\text { Mean } \\
\text { estimate error } \\
\text { (percent) }\end{array}$ & $\begin{array}{c}\text { Standard deviation } \\
\text { of estimate error } \\
\text { (percent) }\end{array}$ \\
\hline$K_{h}$ & $\begin{array}{c}R_{o} \\
\left(R_{o}-R_{w x}\right)\end{array}$ & 21,22 , or 23 & 32 & $3.6-411$ & 55 & 69 \\
$K_{h}$ & $(\mathrm{CH}-186$ data) & 18 or 19 & 6 & $13-79$ & 40 & 25 \\
$K_{h}$ & $\begin{array}{c}R_{o} \\
(\mathrm{CH}-186 \text { data) } \\
R_{o}\end{array}$ & 21 or 22 & 6 & $3.6-56$ & 34 & 18 \\
$K_{h}$ & 21,22 , or 23 & 31 & $3.6-95$ & 43 & 23 \\
\hline
\end{tabular}

resulted in absolute errors ranging from about 2 to 70 percent, and a mean error of 25 percent (table 8). Horizontal hydraulic conductivity was computed at site $\mathrm{CH}-186$ using grain-surface resistivity and bulk resistivity as independent variables (eqs. 18, 19, and 21, 22). Estimates were compared to values of horizontal conductivity determined from permeameter analyses at five cored intervals and from aquifer-test results for the finished well. At three of the five intervals, errors of estimated horizontal hydraulic conductivity based on grain-surface resistivity were less than errors for corresponding values computed using bulk resistivity as the independent variable. These errors ranged from 13 to 79 percent and 4 to 56 percent, respectively (table 9). Results related to the finished well test were similar. Horizontal hydraulic conductivity was also computed at 26 other sites using only bulk resistivity as the independent variable (eqs. 21-23). Absolute error for all sites ranged from about 4 to 400 percent. Mean error was 55 percent (table 9). Absolute error at one site was larger than two standard deviations from the mean absolute error and is considered a statistical outlier (site M12, table 7). When the absolute error for this site was removed, the mean absolute error was 43 percent and the error range was 4 to 95 percent.

\section{SUMMARY AND CONCLUSIONS}

Aquifer bulk resistivity and grain-surface resistivity (inverse of surface conductance) were theoretically related to the horizontal hydraulic conductivity of freshwater aquifers composed of sands and clayey sands. Regression equations were subsequently used to test the theoretical concepts using borehole geophysical, water-quality, and hydraulic characteristics data obtained from more than a hundred well sites in the Southeastern Coastal Plain of the United States. Data were obtained from aquifers composed of sediments ranging in age from Late Cretaceous through middle Eocene. Bulk resistivity was estimated using long-normal (64-inch) and dual induction borehole resistivity logs. Grain-surface resistivity was empirically defined as the difference between bulk resistivity and aquifer water resistivity (computed from specific conductance). Horizontal hydraulic conductivity, as used in this report, is the average aquifer transmissivity per foot of contributing interval and ranged from about 4 to more than $500 \mathrm{ft} / \mathrm{d}$. All paired data were related using the logarithmic regression model $Y=A X^{B}$. All paired data used in regression analyses were considered average values across a specified interval contributing water 
to a finished well. Estimated thicknesses of the contributing intervals ranged from 10 to about $600 \mathrm{ft}$.

Regression analyses indicate a moderate correlation between bulk and grain-surface resistivities and horizontal hydraulic conductivity. The correlation coefficients developed by using all hydraulic conductivity data (105 data pairs) were 70 and 72 percent, respectively. The correlation coefficients of similar analyses using data grouped by the age of water-bearing units (Late Cretaceous, 14 data pairs; Paleocene-early Eocene, 47 data pairs; and middle Eocene, 44 data pairs) ranged from 71 to 80 percent. These results indicate that aquifer bulk resistivity and grain-surface resistivity are useful geoelectrical analogs for the horizontal hydraulic conductivity of clastic, unconsolidated aquifers in the Southeastern United States.

Separate regressions of the resistivity and dissolved-solids concentrations of aquifer water using aquifer bulk resistivity as the independent variable indicated a high degree of correlation between these variables (correlation coefficients of 93 and 88 percent, respectively). An analysis of the relation of dissolved-solids concentration to aquifer water resistivity resulted in an exceptionally high degree of correlation (correlation coefficient of 96 percent). Dissolved-solids concentrations used in these analyses ranged from about 30 to $1,300 \mathrm{mg} / \mathrm{L}$.

An analysis of the relation between horizontal hydraulic conductivity and apparent formation factor (ratio of aquifer bulk resistivity to aquifer water resistivity) resulted in a correlation coefficient of 38 percent. Although formation factor and apparent formation factor had previously been presented as useful geoelectrical analogs to horizontal hydraulic conductivity at a local scale, results of this study indicate that, at a regional scale, little or no significant correlation exists between apparent formation factor and horizontal hydraulic conductivity.

Regression equations presented in this report were applied and tested at a total of 27 sites in 6 Southeastern States. When used as independent variables, aquifer water resistivity and aquifer bulk resistivity were shown to reasonably predict the dissolved-solids concentration of aquifer water (mean absolute errors of about 6 and 20 percent, respectively). Similarly, bulk resistivity was used to estimate aquifer water resistivity (mean error of 25 percent). The absolute error of estimates of horizontal hydraulic conductivity computed using aquifer bulk resistivity as the independent variable ranged from about 4 to 400 percent. Mean absolute error was 55 percent. Elimination of one data point considered to be a statistical outlier improved the mean estimate error to 43 percent with an error range of 4 to 95 percent.

\section{REFERENCES}

Alger, R.P., 1966, Interpretation of electric logs in fresh water wells in unconsolidated formations, in Transactions of 7th Annual Logging Symposium, May 8-11: Tulsa, Okla., Society of Professional Well Log Analysts, p. cc1-cc25.

Alger, R.P., and Harrison, C.W., 1989, Improved freshwater assessment in sand aquifers utilizing geophysical well logs: The Log Analyst, v. 30, no. 1, p. 31-44.

Archie, G.E., 1942, The electrical resistivity log as an aid in determining some reservoir characteristics: American Institute of Mining and Metallurgical Engineers Transactions, v. 146, p. 54-62.

Aucott, W.R., and Newcome, Roy, Jr., 1986, Selected aquifer-test information for the Coastal Plain aquifers of South Carolina: U.S. Geological Survey WaterResources Investigations Report 86-4159, 30 p.

Biella, Giancarlo, Lozej, Alfredo, and Tabacco, Ignacio, 1983, Experimental study of some hydrogeophysical properties of unconsolidated porous media: Ground Water, v. 21, no. 6, p. 741-751.

Brown, D.L., 1971, Techniques for quality-of-water interpretations from calibrated geophysical logs, Atlantic Coastal area: Ground Water, v. 9, no. 4, p. 25-38.

Cahill, J.M., 1982, Hydrology of the low-level radioactive-solid-waste burial site and vicinity near Barnwell, South Carolina: U.S. Geological Survey Open-File Report 82-863, 101 p.

Chapelle, F.H., and McMahon, P.B., 1991, Geochemistry of dissolved inorganic carbon in a Coastal Plain aquifer. 1. Sulfate from confining beds as an oxidant in microbial $\mathrm{CO}_{2}$ production: Journal of Hydrology, v. 127 , no. 1 , p. $85-108$.

Clarke, J.S., Faye, R.E., and Brooks, Rebekah, 1983, Hydrogeology of the Providence aquifer of southwest Georgia: Georgia Geological Survey Hydrologic Atlas 11. 1985, Hydrogeology of the Dublin and Midville aquifer systems of east-central Georgia: Georgia Geological Survey Information Circular 74, 62 p.

Croft, M.G., 1971, A method of calculating permeability from electric logs: U.S. Geological Survey Professional Paper 750-B, p. B265-B269.

Davis, M.E., Sparkes, A.K., and Peacock, B.S., 1983, Results of a test well in the Nanafalia Formation 
near Melvin, Choctaw County, Alabama: U.S. Geological Survey Water-Resources Investigations Report 82-4108, 17 p.

De Wiest, R.J.M., 1965, Geohydrology: John Wiley and Sons, Inc., New York, p. 169-172.

Faye, R.E., and McFadden, K.W., 1986, Hydraulic characteristics of Upper Cretaceous and lower Tertiary clastic aquifers - eastern Alabama, Georgia, and western South Carolina: U.S. Geological Survey Water-Resources Investigations Report 86-4210, $22 \mathrm{p}$.

Ferris, J.G., Knowles, D.B., Brown, R.H., and Stallman, R.W., 1962, Theory of aquifer tests: U.S. Geological Survey Water-Supply Paper 1536-E, $174 \mathrm{p}$.

Guyod, Hubert, 1957, Electric detective investigation of ground-water supplies with electric well logs: Water Well Journal, v. 11, nos. 3, 4.

Hantush, M.S., and Jacob, C.E., 1955, Non-steady radial flow in an infinite leaky aquifer: American Geophysical Union Transactions, v. 36, no. 1, p. 95-100.

Hosman, R.L., Long, A.T., Lambert, T.W., and others, 1968, Tertiary aquifers in the Mississippi embayment: U.S. Geological Survey Professional Paper 448-D, 29 p.

Huntley, David, 1986, Relations between permeability and electrical resistivity in granular aquifers: Ground Water, v. 24, no. 4, p. 466-474.

Jones, P.H., and Buford, T.B., 1951, Electric logging applied to ground-water exploration: Geophysics, v. 16 , no. 1, p. $115-139$.

Jorgensen, D.G., 1989, Using geophysical logs to estimate porosity, water resistivity, and intrinsic permeability: U.S. Geological Survey Water-Supply Paper 2321, 24 p. 1990 , Estimating water quality from geophysical logs: Geophysical Applications for Geo-technical Investigations, ASTM STP 1101, p. 47-64.

Keys, W.S., and MacCary, L.M., 1971, Application of borehole geophysics to water-resources investigations: Techniques of Water-Resources Investigations of the United States Geological Survey, book 2, E1, $126 \mathrm{p}$.

Lee, R.W., 1984, Ground-water quality data from the southeastern Coastal Plain, Mississippi, Alabama, Georgia, South Carolina, and North Carolina: U.S. Geological Survey Open-File Report 84-237, 20 p. 1988, Water-quality maps for the Upper Cretaceous and Lower Tertiary aquifer in the southeastern Coastal Plain of Mississippi, Alabama, Georgia, South Carolina, and southeastern North Carolina: U.S. Geological Survey Water-Resources Investigations Report 86-4116, 2 pl.

Lee, R.W., and Strickland, D.J., 1988, Geochemistry of ground water in Tertiary and Cretaceous sediments of the southeastern Coastal Plain in eastern Georgia, South Carolina, and southeastern North Carolina: Water Resources Research, v. 24, no. 2, p. 291-303.

Mazac, O., Kelly, W.E., and Landa, I., 1985, A hydrogeophysical model for relations between electrical and hydraulic properties of aquifers: Journal of Hydrology, 79, (1-2), p. 1-19.

McMahon, P.B., and Chapelle, F.H., 1991, Geochemistry of dissolved inorganic carbon in a Coastal Plain aquifer. 2. Modeling carbon sources, sinks, and $\delta^{13} \mathrm{C}$ evolution: Journal of Hydrology, v. 127, no. 1, p. 109-135.

Miller, R.L., Bradford, W.L., and Peters, N.E., 1988, Specific conductance - theoretical considerations and application to analytical quality control: U.S. Geological Survey Water-Supply Paper 2311, 16 p.

Muskat, Morris, 1937, The flow of homogeneous fluids through porous media: New York, McGraw-Hill Book Co., Inc., 763 p.

Pettijohn, R.A., 1988, Dissolved-solids concentrations and primary water types, Gulf Coast aquifer systems, south-central United States: U.S. Geological Survey Hydrologic Investigations Atlas HA-706, $2 \mathrm{pl}$.

Pfannkuch, H.O., 1969, On the correlation of electrical conductivity properties of porous systems with viscous flow transport equations, in Proceedings of the First International Symposium on the Fundamentals of Transport Phenomena in Porous Media: Haifa, Israel, International Association of Hydraulic Research, p. 42-54.

Scott, J.C., Law, L.R., and Cobb, R.H., 1984, Hydrology of the Tertiary-Cretaceous Aquifer System in the Vicinity of Fort Rucker Aviation Center, Alabama: U.S. Geological Survey Water-Resources Investigations Report 84-4118, 221 p.

Slack, L.J., and Darden, Daphne, 1991, Summary of aquifer tests in Mississippi, June 1942 through May 1988: U.S. Geological Survey Water-Resources Investigations Report 90-4155, $40 \mathrm{p}$.

Theis, C.V., 1935, The relation between the lowering of the piezometric surface and the rate and duration of discharge of a well using ground-water storage: American Geophysical Union Transactions, v. 16, p. 519-524.

Turcan, A.N., Jr., 1962, Estimating water quality from electric logs: U.S. Geological Survey Professional Paper 450-C, p. C135-C136.

Urish, D.W., 1981, Electrical resistivity-hydraulic conductivity relationships in glacial outwash aquifers: Water Resources Research, v. 17, no. 5, p. 1401-1407.

Worthington, P.F., 1976, Hydrogeophysical equivalence of water salinity, porosity, and matrix conduction in 
arenaceous aquifers: Ground Water, v. 14, no. 4, p. 224-232.

1977, Influence of matrix conduction upon hydrogeophysical relationships in arenaceous aquifers:

Water Resources Research, v. 13, no. 1, p. 87-92.

Worthington, P.F., and Johnson, P.W., 1991, Quantitative evaluation of hydrocarbon saturation in shaly freshwater reservoirs: The Log Analyst, v. 34, no. 4 , p. $358-370$.
Yao An Guo, 1986, Estimation of TDS in sand aquifer water through resistivity log: Ground Water, v. 24, no. 5, p. 598-600.

Zack, Allen, 1977, The occurrence, availability, and chemical quality of ground water, Grand Strand area and surrounding parts of Horry and Georgetown Counties, South Carolina: South Carolina Water Resources Commission Report No. 8, 100 p. 